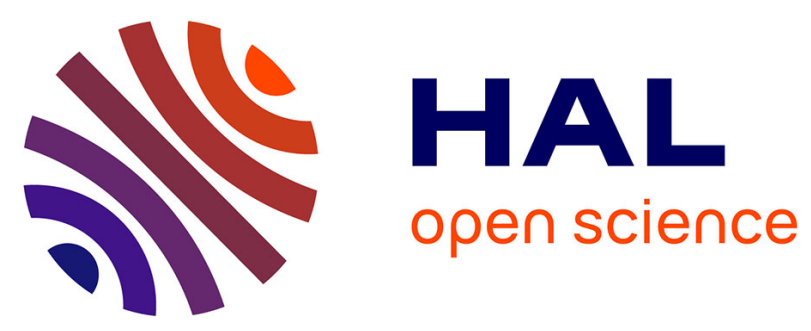

\title{
Introducing a level-set based shape and topology optimization method for the wear of composite materials with geometric constraints
}

Florian Feppon, G Michailidis, M.A. Sidebottom, Grégoire Allaire, B.A. Krick, N Vermaak

\section{To cite this version:}

Florian Feppon, G Michailidis, M.A. Sidebottom, Grégoire Allaire, B.A. Krick, et al.. Introducing a level-set based shape and topology optimization method for the wear of composite materials with geometric constraints. Structural and Multidisciplinary Optimization, 2017, 55 (2), pp.547-568. hal01336301

\section{HAL Id: hal-01336301 \\ https://hal.science/hal-01336301}

Submitted on 22 Jun 2016

HAL is a multi-disciplinary open access archive for the deposit and dissemination of scientific research documents, whether they are published or not. The documents may come from teaching and research institutions in France or abroad, or from public or private research centers.
L'archive ouverte pluridisciplinaire HAL, est destinée au dépôt et à la diffusion de documents scientifiques de niveau recherche, publiés ou non, émanant des établissements d'enseignement et de recherche français ou étrangers, des laboratoires publics ou privés. 


\title{
Introducing a level-set based shape and topology optimization method for the wear of composite materials with geometric constraints
}

\author{
F. Feppon - G. Michailidis - M.A. \\ Sidebottom • G. Allaire • B.A. Krick • N. \\ Vermaak
}

The final publication is available at Springer via http://dx.doi.org/10.1007/s00158-016-1512-4

\begin{abstract}
The wear of materials continues to be a limiting factor in the lifetime and performance of mechanical systems with sliding surfaces. As the demand for low wear materials grows so does the need for models and methods to systematically optimize tribological systems. Elastic foundation models offer a simplified framework to study the wear of multimaterial composites subject to abrasive sliding. Previously, the evolving wear profile has been shown to converge to a steadystate that is characterized by a time-independent elliptic equation. In this article, the steady-state formulation is generalized and integrated with shape optimization to improve the wear performance of bi-material composites. Both macroscopic structures and periodic material microstructures are considered. Several common tribological objectives for systems undergoing wear are identified and mathematically formalized with shape derivatives. These include (i) achieving a planar wear surface from multimaterial composites and (ii) minimizing the run-in volume of material lost before steady-state wear is achieved. A level-set based topology optimization algorithm that incorporates a novel constraint on the level-set function is presented. In particular, a new scheme is developed to update material interfaces; the scheme (i) conveniently enforces volume constraints at each iteration, (ii)
\end{abstract}

F. Feppon

École polytechnique, 91128 Palaiseau, France

E-mail: florian.feppon@polytechnique.edu

G. Michailidis

SIMaP-Université de Grenoble, INPG, 3800 Grenoble, France

M.A. Sidebottom

Mechanical Engineering and Mechanics, Lehigh University, Bethlehem PA, USA

G. Allaire

CMAP, École polytechnique, CNRS UMR 7641, 91128 Palaiseau, France

B.A. Krick

Mechanical Engineering and Mechanics, Lehigh University, Bethlehem PA, USA

N. Vermaak

Mechanical Engineering and Mechanics, Lehigh University, Bethlehem PA, USA 
controls the complexity of design features using perimeter penalization, and (iii) nucleates holes or inclusions with the topological gradient. The broad applicability of the proposed formulation for problems beyond wear is discussed, especially for problems where convenient control of the complexity of geometric features is desired.

Keywords Wear · tribology · steady-state · geometric constraints · shape optimization · level-set method · perimeter penalization · topological gradient

\section{Introduction}

The design of wear surfaces is an interdisciplinary endeavor often involving mechanics, materials, and chemistry. Wear is a facet of tribological systems that refers to the gradual removal of a material from surfaces of solids subject to contact and sliding (Hatchett (1803); Archard (1953); Archard and Hirst (1956)). Abrasive sliding wear is the removal of material from a surface that is sliding against another surface, typically harder materials against softer materials (see Figure 1p) (Rabinowicz and Mutis (1965); Lancaster (1969); Sin et al (1979); Khruschov (1974); Rabinowicz et al (1961)). Friction is commonly considered an instantaneous pro-

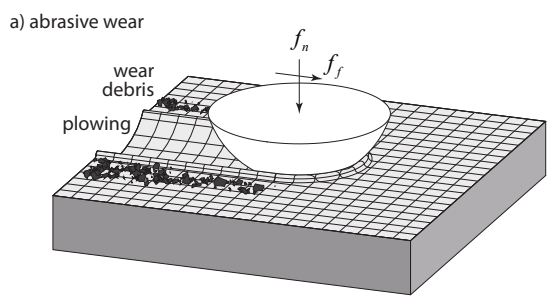

b) wear rate, $\mathrm{K}$ :

volume lost $(V)$ per normal load $\left(F_{N}\right)$ per distance of sliding $(d)$

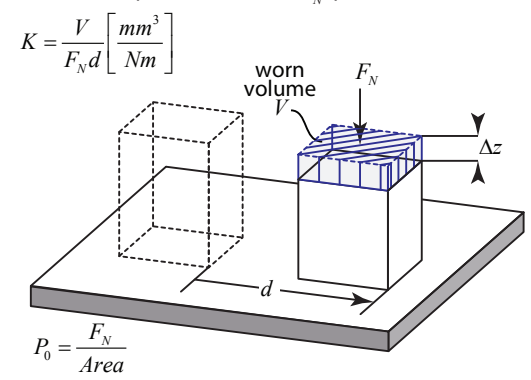

Fig. 1: (a) Schematic of abrasive wear between a hard asperity and a sample. (b) Illustration defining Archard wear rates.

cess (and frictional losses are of particular interest in engineering systems). Wear of tribological systems is caused by continuous frictional interactions between two surfaces in contact. These interactions lead to damage of a material's microstructure near the surface. This damage accumulates until portions of the material's 
surface are removed, which over time can cause failure at the contact interface. In this context, friction and contact models are used to optimize system properties and characteristics such as fracture resistance, frictional energy loss, and heat generation with respect to specific and instantaneous loading conditions (Hilding et al (1995); Challis et al (2008); Schmidt and Hattel (2008)). In contrast, optimizing wear performance is critical to improve the durability of systems subjected to continuous abrasion.

Predicting the wear performance of a system, such as the topographical evolution of a wearing surface or the volume of material lost during wear is of practical interest in many industrial applications. For example, these predictions can be used in the mechanical design of components and to estimate service life (Põdra and Andersson (1997, 1999a b); Kim et al (2005); Mukras et al (2010, 2009); Lengiewicz and Stupkiewicz (2013); Fregly et al (2005); Chongyi et al (2010); Telliskivi (2004); Sawyer et al (2014)). Most wear models involve mechanics-based relations between geometry, pressure, and material wear properties for a given configuration of materials. Every material system has some resistance to material removal that is described by a parameter called the wear rate (Figure 1 b). This wear rate is a system parameter that depends on the combinations of constituent materials, environmental effects, sliding conditions, etc. (Blau (1997); Sawyer et al (2014); Zum Gahr (1987)). Wear rates can be experimentally measured for materials using several standard procedures (ASTM Standard D3702-94 (1974/2014); ASTM Standard G77-05 (1983/2010); ASTM Standard G99-05 (1990/2010); Archard and Hirst (1956); Colbert et al (2011); Schmitz et al (2004); Rowe et al (2014); Erickson. et al (2015)). Depending on a model's level of complexity, these wear rates may be further related to material hardness, ductility, surface chemistry, and adhesion (Zum Gahr (1987)).

To date, many techniques have been proposed to simulate wear that range from molecular dynamics to simple analytical models (Blanchet (1997); Sawyer (2001); Dickrell and Sawyer (2004); Telliskivi (2004); Põdra and Andersson (1999b); Mukras et al (2009); Johansson (1994); Dickrell et al (2003); Kim et al (2005); Rowe et al (2014); Sawyer (2004); Sawyer et al (2014); Jang et al (2007)). Most often the approach consists in simulating the wear evolution of a surface with iterative schemes by assuming a relation between material removal rates and local contact pressures. Each iteration evaluates the pressure distribution on the contact surface and with this pressure, updates the geometry of the contact. As a consequence, the design of tribological systems has primarily been studied with these numerical iterative schemes. The absence of explicit, generic and continuous formulations as well as the computational cost associated with the use of these iterative procedures have prevented modern topology optimization and control techniques from being systematically applied to wearing systems (Feppon et al $(2015))$. Formally optimizing wear performance is the focus of this article. In particular, sliding abrasive wear will be considered because simplified mechanical models have already been developed and experimentally validated for this kind of wear (Põdra and Andersson (1999b); Fregly et al (2005); Kim et al (2005); Blanchet (1997); Dickrell et al (2003); Sawyer (2004); Rowe et al (2014)). Predicting wear performance remains an open domain, rich with challenges for industries aiming to design systems with improved or target wear characteristics (Tankala and Kapoor (2001); Barron (1982); Prasad (2000)). Few attempts have been made to improve the wear performance of systems with mathematical optimization (Páczelt and Mróz (2005); Willing and Kim (2009); 
Markine et al (2007); Choi et al (2013)). These works have focused on optimizing indirect factors, such as contact forces or the pressure distributions for which contact optimization theory is available (Hilding et al (1995); Myśliński (2012); Strömberg and Klarbring (2010); Neittaanmäki et al (1988)), without optimizing for wear and its long-term effects.

In Feppon et al (2015), it has been shown that the well-established framework offered by elastic foundation models (Kerr (1964); Pasternak (1954)) allows one to propose a continuous and mathematically well-posed formulation for the abrasive sliding wear of composites or heterogeneous materials. Foundation models simplify the contact mechanics analysis by postulating explicit relationships between surface topography and pressure distributions (Figure 2). While the contact mechanics analysis is simplified, they are nevertheless commonly accepted and experimentally validated models for calculating contact pressure distributions (Põdra and Andersson (1999b); Kim et al (2005); Fregly et al (2005); Sawyer (2001)). Experimentally, it is well-known that, under a constant average pressure

a) model configuration

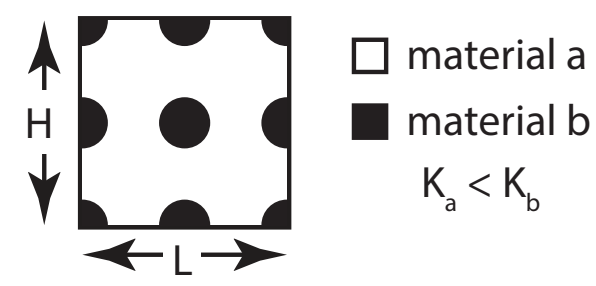

b) wear iterations (pressure and topography)

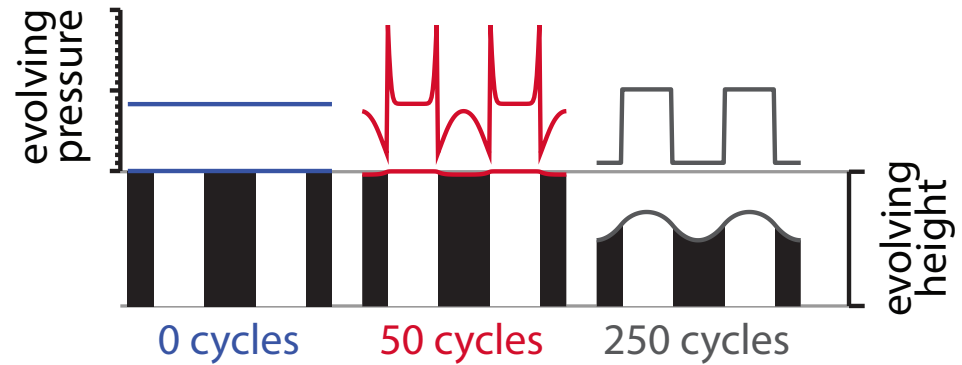

Fig. 2: Representation of the evolution of topography and pressure distribution for an initially flat bi-material surface. a) Initial distribution of two materials (material "a" and material "b") along the sliding surface. b) Example evolution of contact pressure and surface topography for a bi-material surface after 0,50 , and 250 sliding cycles.

load, the wear of an initially flat, heterogeneous distribution of materials may lead to a non-planar worn surface. Eventually, the recessing wear profile reaches a steady-state that continues to recess at a constant rate (Rowe et al (2014); Lee et al (2002); Hilding et al (2001); Axén and Jacobson (1994)). This transient pe- 
riod before steady-state is reached is commonly called "run-in" wear. Foundation models mathematically recover this run-in characteristic through the existence of a corresponding asymptotic expansion of the wearing profile (Feppon et al $(2015))$. Remarkably, the limit steady-state profile can be determined explicitly from the solution of a time-independent, elliptic partial differential equation. This facilitates the possibility to apply modern topology optimization techniques with the goal of improving the wear performance of tribological systems (Bendsøe and Sigmund (2003); Allaire (2007a); Eschenauer and Olhoff (2001); Vermaak et al (2014)). The formulation proposed herein is also compatible with other elliptic models, which could be used for example, to explore multi-physics wear optimization.

In this article, for the first time, a topology optimization framework is proposed for wear applications. The focus will be on linear abrasive sliding wear for which sliding direction is fixed. A generalization of the wear model employed in Rowe et al (2014); Feppon et al (2015); Sawyer (2004), that is mathematically suitable for topology optimization, is introduced in Section 2. Governing equations for the corresponding steady-state solution are explained. A relaxation result is proven in Section 3 that gives insight into the relationships between wear-optimal material distributions, material volume fractions, and material microstructures. The limitations of the underlying foundation models and the consequences for the topology optimization of macroscopic structures and periodic material microstructures are discussed. Objective functions for common wear performance metrics and their shape derivatives are calculated. Minimizing these objectives with level-set based topology optimization methods (Osher and Sethian (1988); Allaire et al (2004); Wang et al (2003) ) is considered in Section 4 for 2D multimaterial composites. This article also presents a new way to control the complexity of design features that is broadly applicable beyond wear and tribological problems. The level-set based topology optimization method presented uses a novel constraint on the level-set function (Section 5). This results in an evolution equation that can be used to simultaneously update the geometry (by moving the bi-material interface) and the topology (by nucleating holes or inclusions). It is also shown that the simplicity of the evolution equation allows one to both easily impose equality volume constraints at each iteration, and to control the complexity of design features through perimeter penalization. Numerical results and optimal material distributions are presented and discussed in Section 6 .

\section{Time-independent steady-state model for abrasive sliding wear}

\subsection{Governing equation for an evolving wear profile}

In the following, a multimaterial composite system, $\Omega$, subject to abrasive sliding wear is assumed. The resistance to material removal in each point of the domain, $x \in \Omega$, is characterized by a wear rate coefficient, $K(x)$. Mathematically, $K$ may be any measurable function satisfying $K_{\min } \leqslant K \leqslant K_{\max }$ for some $K_{\min }, K_{\max }>0$. For a multimaterial distribution, $K$ is a piecewise constant function in each of the material phases (Figure 3). The wear profile height, $z(s, x)$, depends on the $2 \mathrm{D}$ position, $x$, and on the distance of abrasive sliding, $s$. Classically in wear prediction, Archard's law (Archard and Hirst (1956)) states that the layer of material, dz, to be removed after a distance of sliding, $\mathrm{d} s$, is proportional to the material wear rate and to the pressure load, $p$, at each point. As wear is assumed to be a continuous 


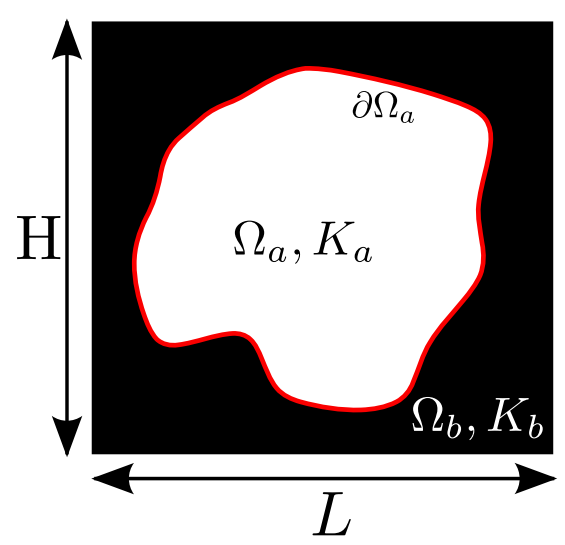

Fig. 3: Schematic of the bi-material distribution

process, the following relationship for the wearing profile height, $z(s, x)$, can be derived (Feppon et al (2015)):

$$
\frac{\partial z}{\partial s}=-p K
$$

In Rowe et al (2014); Feppon et al (2015); Sidebottom et al (2015), a Pasternak elastic foundation model (Kerr (1964); Pasternak (1954)) was assumed to characterize the pressure distribution, $p$, with a constant average pressure constraint Feppon et al (2015)), $<p>=\frac{1}{|\Omega|} \int_{\Omega} p \mathrm{~d} x=P_{0}$ :

$$
p=P_{0}+k_{s}(z-<z>)-k_{g}(\Delta z-<\Delta z>),
$$

where $\Delta z=\frac{\partial^{2} z}{\partial x^{2}}+\frac{\partial^{2} z}{\partial y^{2}}$ is the Laplacian of $z$, and $k_{s}, k_{g}>0$ are two elastic foundation parameters. Elastic foundation models assume that wear contact can be described in the context of a bed of spring elements (with rigidity $k_{s}$ ) that are coupled with a bending beam element (characterized by a tension constant $k_{g}$, Figure 4). Thus, the contact pressure distribution is related to the deflection of each spring element and to the local curvature, $\Delta z(\operatorname{Kerr}(1964) ;$ Pasternak $(1954)$; Sawyer (2004)).

Before discussing the boundary conditions for $z$, the solution of equation (2), it should be noted that the approach presented is general and can accommodate most types of boundary conditions. This includes even more general affine differential relationships than equation (2) between the profile height, $z$, and the pressure distribution, $p$. For example, a more general relation is found by introducing a weak formulation of the equations, namely a bilinear form $a$ and a linear form $l$, defined on $H^{1}(\Omega)$, the classical Sobolev space of bounded energy functions (see Allaire $(2007 \mathrm{~b})$ ). This allows one to express the dependence between the profile height, $z \in H^{\perp}(\Omega)$, and the pressure, $p \in L^{2}(\Omega)$, in a weak form as:

$$
\forall v \in H^{1}(\Omega), \int_{\Omega} p v \mathrm{~d} x=-l(v)+a(z, v)
$$


contact mechanics framework:

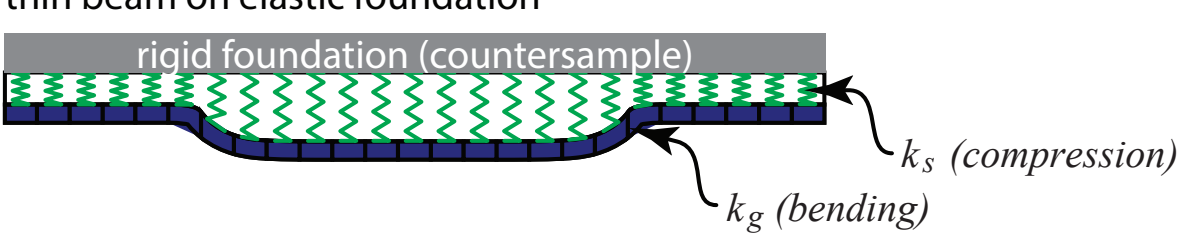

local contact pressure, $\mathrm{p}$

$p=P_{0}+k_{S}(z-<z>)-k_{g}(\Delta z-<\Delta z>)$

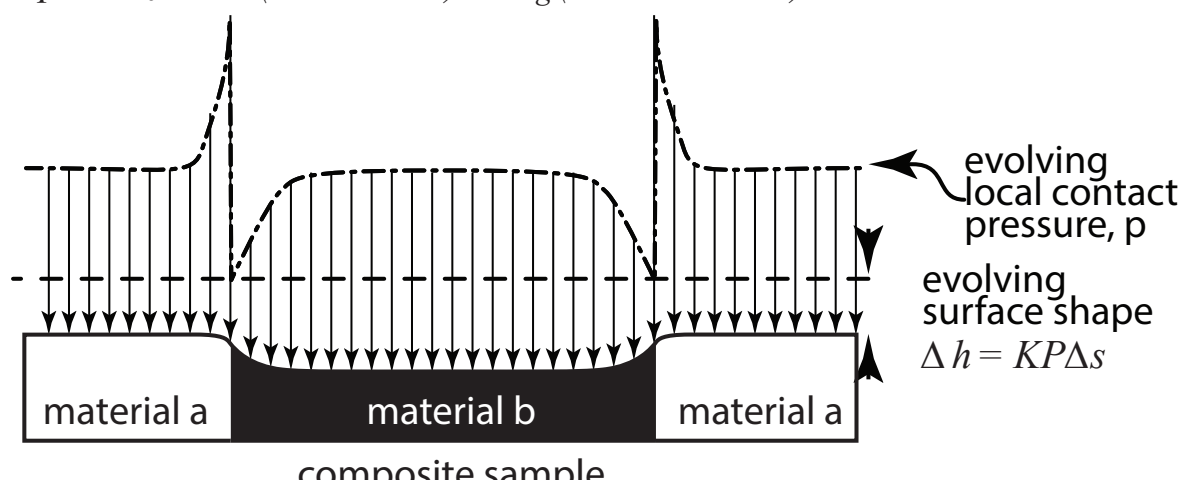

composite sample

Fig. 4: Physical description of the numerical wear model. The Pasternak elastic foundation model is composed of spring elements that are coupled with bending beam elements; the corresponding parameters are $k_{s}$ and $k_{g}$, respectively. The pressure applied at each node is a function of the deflection of the spring element and the local curvature.

The average pressure constraint, $\langle p\rangle=P_{0}$, that must be satisfied by any profile, $z \in H^{1}(\Omega)$, is ensured by requiring that the bilinear form, $a$, is null on the constants and $l(1)=-|\Omega| P_{0}$. The time dependent equation describing the evolution of the profile, $z$, is obtained by combining equations (1) and (3) (see Feppon et al (2015)), and is written in the weak form (Allaire $(2007 \mathrm{~b}))$ as:

$$
\begin{aligned}
& \text { Find } z \in L^{2}(] 0, T\left[; H^{1}(\Omega)\right) \cap \mathcal{C}\left([0, T] ; L^{2}(\Omega)\right) \text { such that } \\
& \qquad s \geqslant 0, \forall v \in H^{1}(\Omega), \frac{\mathrm{d}}{\mathrm{d} s} \int_{\Omega} \frac{z v}{K} \mathrm{~d} x+a(z, v)=l(v) .
\end{aligned}
$$

Of course, equation (4) must be supplemented with some initial conditions, $z_{0} \in$ $L^{2}(\Omega)$, such that $z(0, \cdot)=z_{0}$. For physical applications, this article considers an extension of the Pasternak foundation model by introducing a reference pressure, $f \in L^{2}(\Omega)$, satisfying:

$$
<f>=P_{0},
$$

and by replacing equation with:

$$
p=f+k_{s}(z-<z>)-k_{g}(\Delta z-<\Delta z>) .
$$


The reference pressure profile in (Feppon et al $(2015)$; Rowe et al $(2014)$; Sawyer (2004)) and equation (2) was assumed to be flat, that is $f=P_{0}$. The introduction of a non-constant parameter, $f$, can be motivated by the fact that the pressure distribution for a flat profile, $z=0$, may not be uniform. Indeed, flat punch contact theory predicts that a non-uniform pressure profile (with elevated values at the edges) results from the contact between a rigid flat body on a Pasternak foundation (Ciavarella et al (1998); Marzeda et al (2001)) (see Figure 5). The further relevance of the introduction of this parameter will be discussed in Section 6 .

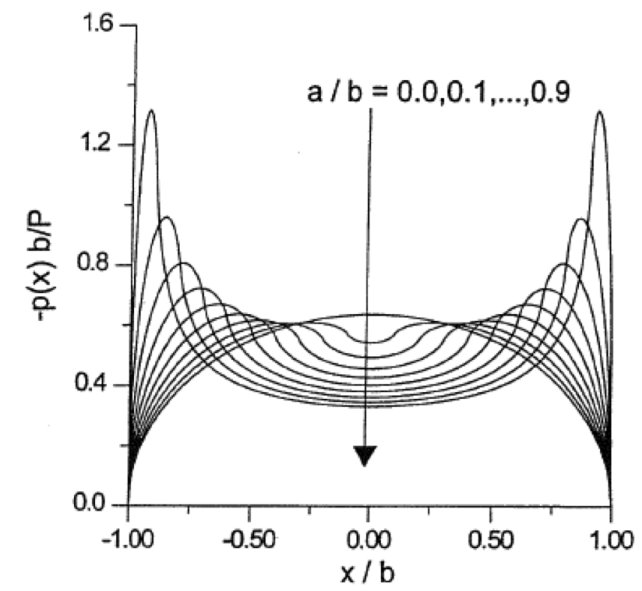

Fig. 5: Theoretical flat punch pressure profile (from Ciavarella et al (1998)).

Boundary conditions in addition to equation (6) are required to ensure the well-posedness of the model. In Rowe et al (2014); Feppon et al (2015), periodic boundary conditions were considered by tribologists because they were both conveniently implemented in iterative schemes and compatible with the study of multimaterial composites on a periodic, unit-cell basis. For these reasons, they will be used for the topology optimization results presented in Section 6. As far as the authors are aware, no other boundary conditions have been proposed in the available wear literature for these elastic foundation models. The analysis performed here shows that the following Fourier boundary conditions for the profile, $z$, are also mathematically suitable:

$$
k_{g} \frac{\partial z}{\partial n}+\beta(z-<z>)=g
$$

where $\beta$ is a positive bounded function, $g$ represents a load on the boundary $\partial \Omega$, and $n$ is the outward normal to the boundary $\partial \Omega$. Note that in this more general framework, Dirichlet boundary conditions $(z=<z>$ ) are found by taking the limit, $\beta \rightarrow+\infty$. With this set of boundary conditions, the bilinear form $a$ and the 
linear form $l$ of equation (3) are given by:

$$
\begin{array}{r}
a(u, v)=\int_{\Omega} k_{s}(u-<u>)(v-<v>)+k_{g} \nabla u \cdot \nabla v \mathrm{~d} x \\
+\beta \int_{\partial \Omega}(u-<u>)(v-<v>) \mathrm{d} s, \\
l(v)=-\int_{\Omega} f v \mathrm{~d} x+\int_{\partial \Omega} g(v-<v>) \mathrm{d} s .
\end{array}
$$

Periodic boundary conditions are also included in this analysis, as all of the expressions obtained in this paper with homogeneous Neumann boundary conditions $\left(\frac{\partial z}{\partial n}=0\right.$ on the boundary, $\left.\partial \Omega\right)$ can also be used without modification, for the case of periodic boundary conditions. This is because both boundary conditions share identical variational formulations. Indeed in both cases, the average local change in height is null, that is $\langle\Delta z\rangle=\frac{1}{|\Omega|} \int_{\partial \Omega} \frac{\partial z}{\partial n} \mathrm{~d} s=0$.

Finally, the weak time-dependent system of equations (4) can be written in the strong form to characterize the evolving wear profile, $z$ :

$$
\left\{\begin{aligned}
\frac{1}{K} \frac{\partial z}{\partial s}+k_{s}(z-<z>)-k_{g}(\Delta z-<\Delta z>) & =-f \text { in } \Omega \\
k_{g} \frac{\partial z}{\partial n}+\beta(z-<z>) & =g \text { on } \partial \Omega .
\end{aligned}\right.
$$

\subsection{A steady-state time-independent formulation}

It has been shown in Feppon et al (2015) that the general variational formulation expressed in equation (4) allows one to derive a time-independent governing set of equations. The following asymptotic expansion for a wear profile, $z$, was established:

Proposition 1 Assume that the bilinear form a, defined by (8), satisfies the following hypotheses:

(i) a is coercive on the space $V_{K}=\left\{v \in H^{1}(\Omega) \mid \int_{\Omega} \frac{v}{K} \mathrm{~d} x=0\right\}$

(ii) a vanishes for constants, namely $a(1, v)=a(v, 1)=0$ for any $v \in H^{1}(\Omega)$

Then, there exists a unique $u \in V_{K}$ solution of:

$$
\forall v \in V_{K}, \quad a(u, v)=l(v),
$$

or equivalently, assuming $l(1)=-P_{0}|\Omega|, u \in V_{K}$ is the unique solution of

$$
\forall v \in H^{1}(\Omega), \quad a(u, v)=l(v)+P_{0} \int_{\Omega} \frac{<K^{-1}>^{-1}}{K} v \mathrm{~d} x .
$$

Similarly, equation (4) admits a unique solution $z$, satisfying the initial condition, $z(0, \cdot)=z_{0} \in L^{2}(\Omega)$. Furthermore, there exists a constant $\lambda>0$, such that the following asymptotic expansion holds true:

$$
\forall s \geqslant 0, z(s, x)=-P_{0}\left\langle K^{-1}>^{-1} s+\left\langle\frac{\left\langle K^{-1}>^{-1}\right.}{K} z_{0}\right\rangle+u(x)+g(s, x),\right.
$$


with $g$ satisfying $\left\langle\frac{g(s, \cdot)}{K}\right\rangle=0$ for any $s \geqslant 0$ and

$$
\forall s \geqslant 0,\|g(s, \cdot)\|_{K} \leqslant e^{-\lambda s}\left\|z_{0}-\left\langle\frac{<K^{-1}>^{-1}}{K} z_{0}\right\rangle-u\right\|_{K}
$$

where $\|\cdot\|_{K}$ is the equivalent quadratic norm defined for all $v \in L^{2}(\Omega)$ by $\|v\|_{K}=$ $\sqrt{\int_{\Omega} \frac{v^{2}}{K} \mathrm{~d} x}$

Equation 12 can be physically interpreted as the fact that the asymptotic pressure, $p_{\text {steady }}$, must be proportional to the inverse of the wear rate distribution, $K$, that is (see equation (3)):

$$
p_{\text {steady }}=P_{0} \frac{<K^{-1}>^{-1}}{K} .
$$

This result is of practical importance for the analysis of wear profiles. The asymptotic expansion (13) shows that the wear profile, $z$, is an affine function of the sliding distance, $s$, up to a remainder term, $g$, which is exponentially decaying with $s$. In other words, for large sliding distance $s$, the wear profile $z$ admits a rigid profile, $u$, which recesses at the equivalent wear rate, $\left\langle K^{-1}\right\rangle^{-1}$. To first order, equation (13) proves that the total loss in height is asymptotically proportional to the pressure load, $P_{0}$, the sliding distance, $s$, and the equivalent wear rate $\left\langle K^{-1}\right\rangle^{-1}$. The expression for the wear rate $\left(\left\langle K^{-1}\right\rangle^{-1}\right)$ is also the "inverse rule of mixtures" or "harmonic mean" from classical composite theory (Han and Blanchet (1997); Lee et al (2002); Axén and Jacobson (1994)). For example, in the case of a system with two materials (A and B) that have wear rates $K_{a}$ and $K_{b}$, and an area fraction $A^{*}$ of material $A$, the rule of mixtures is usually written (Rowe et al (2014); Hovis et al (1986); Han and Blanchet (1997)):

$$
<K^{-1}>^{-1}=\frac{K_{a}}{A^{*}+\frac{K_{a}}{K_{b}}\left(1-A^{*}\right)} .
$$

In the physical case where the bilinear and the linear forms, $a$ and $l$, are given by equations (8) and (9), with $f$ satisfying $\langle f\rangle=P_{0}$, the steady-state profile, $u$, defined by equation $\sqrt{12}$, can be rewritten as the unique solution in $H^{1}(\Omega)$ of the differential system:

$$
\left\{\begin{array}{rlrl}
k_{s}(u-<u>)-k_{g}(\Delta u-<\Delta u>) & =-f+P_{0} \frac{<K^{-1}>^{-1}}{K} \text { in } \Omega \\
k_{g} \frac{\partial u}{\partial n}+\beta(u-<u>) & =g & \text { on } \partial \Omega \\
\left\langle\frac{u}{K}\right\rangle & =0 .
\end{array}\right.
$$

This governing equation (17) is a versatile formulation that is convenient to study the long-term wear of multimaterial composites. In addition to the substantial computational gain discussed in Feppon et al (2015), this formulation is also well suited for optimization; this is in contrast to traditional iterative schemes in the wear literature. The key factor is the introduction of explicit continuous governing equations. Directly considering the steady-state wear profile, $u$, instead of the 
recessing time-dependent wear profile, $z$, allows one to formulate wear optimization problems that have the advantage of depending only on the distribution of material, $K$, and not on an arbitrarily-fixed sliding distance (a parameter that can be interpreted as a pseudo-time) or on an initial profile, $z_{0}$. The solution of the system (17) can be computed in practice by using the following proposition.

Proposition 2 Let $f_{1} \in L^{2}(\Omega)$ and $g_{1} \in L^{2}(\partial \Omega)$ be two functions such that $\left\langle f_{1}\right\rangle=$ 0 . Let $w_{0}$ and $w_{1}$ be the unique solutions in $H^{1}(\Omega)$ of:

$$
\begin{aligned}
& \left\{\begin{array}{l}
k_{s} w_{0}-k_{g} \Delta w_{0}=f_{1} \text { in } \Omega \\
k_{g} \frac{\partial w_{0}}{\partial n}+\beta w_{0}=g_{1} \text { on } \partial \Omega
\end{array}\right. \\
& \left\{\begin{array}{l}
k_{s} w_{1}-k_{g} \Delta w_{1}=1 \text { in } \Omega \\
k_{g} \frac{\partial w_{1}}{\partial n}+\beta w_{1}=0 \text { on } \partial \Omega .
\end{array}\right.
\end{aligned}
$$

Then the unique solution, $u_{0}$, in $H^{1}(\Omega)$ of the system:

$$
\left\{\begin{aligned}
k_{s}\left(u_{0}-<u_{0}>\right)-k_{g}\left(\Delta u_{0}-<\Delta u_{0}>\right) & =f_{1} \text { in } \Omega \\
k_{g} \frac{\partial u_{0}}{\partial n}+\beta\left(u_{0}-<u_{0}>\right) & =g_{1} \text { on } \partial \Omega \\
<u_{0}> & =0,
\end{aligned}\right.
$$

is given by:

$$
u_{0}=w_{0}-<w_{0}>\frac{w_{1}}{<w_{1}>} .
$$

Proof The result is easily obtained by checking that the variational formulation of equation 20 , found using Green's identity, and that of $w_{0}-<w_{0}>\frac{w_{1}}{<w_{1}>}$, are identical.

Equations (18) and $(19)$ can be solved using a standard finite-element solver such as FreeFem $++($ Hecht $(2012))$. For the remainder of this work:

$$
f_{1}=-f+P_{0} \frac{<K^{-1}>^{-1}}{K} \text { and } g_{1}=g .
$$

Then, computing the solution, $u_{0}$, of equation 20 allows one to determine the steady-state profile, $u$ (equation (17)), from the relation:

$$
u=u_{0}-\left\langle\frac{<K^{-1}>^{-1}}{K} u_{0}\right\rangle .
$$

\section{A relaxation result in the context of topology optimization}

In the following, the bilinear and linear forms $a$ and $l$ are assumed to be independent of the material distribution $K$, and $a$ is also assumed to be coercive on the space $V_{1}=\left\{v \in H^{1}(\Omega) \mid\langle v\rangle=0\right\}$. This is the case in previous works that use the Pasternak foundation model (Rowe et al (2014); Sidebottom et al (2015)) and in equation (2), but it is also true for the extended model (equations 5 50 ) considered here. For any wear-rate coefficient, $K \in L^{\infty}(\Omega)$, satisfying $0<K_{\min } \leqslant K \leqslant K_{\max }$, the notation $u_{0}(K)=u(K)-<u(K)>$ is used where $u(K)$ denotes the solution of 
equation (12). Equivalently, $u_{0}(K)$ is the unique solution in $V_{1}$ of the variational problem:

$$
\forall v \in V_{1}, \quad a\left(u_{0}(K), v\right)=l(v)+P_{0} J(K)(v),
$$

where the linear form $J(K)$ depends on the wear-rate coefficient, $K$, and is defined by:

$$
\forall v \in L^{2}(\Omega), \quad J(K)(v)=\int_{\Omega} \frac{<K^{-1}>^{-1}}{K} v \mathrm{~d} x .
$$

It was shown in the previous section that the steady-state, $u(K)$, defined by equation $(12)$, is related to $u_{0}(K)$ by means of an additive constant (see equation (23)). For this reason, the term "steady-state" will be used in the following to designate both $u(K) \in V_{K}$ as well as $u_{0}(K) \in V_{1}$.

Topology optimization is now considered in the framework of Proposition 1. Improving the wear performance of a given in-plane configuration of materials, $K$, is accomplished by optimizing the corresponding steady-state wear profile, $u_{0}(K)$. In the following, the system is assumed to be comprised of two materials, $A$ and $B$, with wear rates $K_{a}, K_{b}$. There is a sub-domain, $\Omega_{a} \subset \Omega$, filled with material $A$, and the complementary, $\Omega \backslash \Omega_{a}$, is filled with material $B$ (Figure 3 ). This means that the coefficient, $K\left(\Omega_{a}\right)$, is given by:

$$
K\left(\Omega_{a}\right)=\left(K_{a}-K_{b}\right) \mathbf{1}_{\Omega_{a}}+K_{b},
$$

where $\mathbf{1}_{\Omega_{a}}$ is the characteristic function of the domain $\Omega_{a}$.

A general optimization problem for wear with volume constraints is expressed as:

$$
\inf _{\Omega_{a}, \theta^{-}|\Omega| \leqslant\left|\Omega_{a}\right| \leqslant \theta^{+}|\Omega|} \mathcal{J}\left(u_{0}\left(K\left(\Omega_{a}\right)\right)\right),
$$

where $\mathcal{J}$ is assumed to be a continuous function from $H^{1}(\Omega)$ in $\mathbb{R}$, bounded from below, and $0 \leq \theta^{-} \leqslant \theta^{+} \leq 1$ are two parameters that allow for equality or inequality volume constraints to be considered.

A relaxed formulation of equation (27) is found by replacing the characteristic function, $\mathbf{1}_{\Omega_{a}}$, by a density function, $\theta \in L^{2}(\Omega,[0,1])($ Allaire $(2002))$. The function $\theta$ can be interpreted as a density ratio between materials $A$ and $B$. The only subtle point is that the variable coefficient $K\left(\Omega_{a}\right)$ is replaced by its harmonic mean, $K(\theta)$, defined by:

$$
\frac{1}{K(\theta)}=\frac{\theta}{K_{a}}+\frac{1-\theta}{K_{b}} \text {. }
$$

The relaxation of equation 27] is then:

$$
\inf _{\theta \in L^{2}(\Omega), \theta^{-} \leqslant<\theta>\leqslant \theta^{+}} \mathcal{J}\left(u_{0}(K(\theta))\right),
$$

where $u_{0}(K(\theta))$ is a solution of equation (24) with the linear form $J(K)$ defined by equation (25) and $K=K(\theta)$. The justification of such a relaxed formulation is given by the following result which states that, in some sense, equation (29) is equivalent to the initial optimization problem (equation 27).

Proposition 3 The formulation of equation (29) is the relaxation of the shape optimization problem (27) in the following sense: 
(i) There exists an optimal density $\theta^{*} \in L^{2}(\Omega,[0,1])$ which minimizes equation (29).

(ii) For any minimizing sequence of sub-domains $\left(\Omega_{a}^{n}\right)$ for the problem $\sqrt{27}$, , the associated sequence of characteristic functions $\mathbf{1}_{\Omega_{a}^{n}}$ converges weakly in $L^{2}(\Omega)$, up to a subsequence, to a minimizer $\theta^{*}$ for the problem (29).

(iii) Any minimizer of (29) is attained as the limit of a minimizing sequence of subdomains $\left(\Omega_{a}^{n}\right)$ for $\left.\sqrt{27}\right)$.

In particular, the optimization problem (27) and its relaxation (29) share the same minimal value:

$$
\min _{\theta \in L^{2}(\Omega), \theta^{-} \leqslant<\theta>\leqslant \theta^{+}} \mathcal{J}\left(u_{0}(K(\theta))\right)=\inf _{\Omega_{a}, \theta^{-}|\Omega| \leqslant\left|\Omega_{a}\right| \leqslant \theta^{+}|\Omega|} \mathcal{J}\left(u_{0}\left(K\left(\Omega_{a}\right)\right)\right) .
$$

Proof The proof requires only classical arguments of weak convergence in Hilbert spaces and does not rely on more involved arguments of homogenization theory (see e.g. Allaire $(2002))$. This simple proof is possible because the solution, $u_{0}\left(K\left(\Omega_{a}\right)\right)$, of equation (24) depends on the sub-domain, $\Omega_{a}$, only through the linear form, $J\left(K\left(\Omega_{a}\right)\right)$, in the right hand side of the equation, which itself depends on the characteristic function $\mathbf{1}_{\Omega_{a}}$. Since the sequence of characteristic functions, $\mathbf{1}_{\Omega_{a}^{n}}$, is bounded in $L^{2}(\Omega)$, one can extract a subsequence which converges weakly to a limit, $\theta$, in $L^{2}(\Omega)$. Therefore, for this subsequence, the average $\left\langle K\left(\Omega_{a}^{n}\right)^{-1}>\right.$ converges to $\left\langle K(\theta)^{-1}>\right.$ and:

$$
\forall v \in L^{2}(\Omega), \quad \lim _{n \rightarrow+\infty} \int_{\Omega} \frac{v}{K\left(\Omega_{a}^{n}\right)} \mathrm{d} x=\int_{\Omega} \frac{v}{K(\theta)} \mathrm{d} x .
$$

As a consequence it can be shown, by taking the limit in the variational formulation (equation 24, that the sequence of solutions $u_{0}\left(K\left(\Omega_{a}^{n}\right)\right)$ is also converging strongly in $H^{1}(\Omega)$ to the solution $u(K(\theta))$. Indeed, the convergence:

$$
\forall v \in V^{1}, \quad \lim _{n \rightarrow+\infty} J\left(K\left(\Omega_{a}^{n}\right)\right)(v)=J(K(\theta))(v),
$$

together with the coercive character of $a$, implies the weak convergence in $H^{1}(\Omega)$ of $u_{0}\left(K\left(\Omega_{a}^{n}\right)\right)$ towards the limit $u_{0}(K(\theta))$. Then, due to the Rellich compactness theorem, the convergence holds strongly in $L^{2}(\Omega)$. Eventually, using equation (24) with the test function $v=u_{0}\left(K\left(\Omega_{a}^{n}\right)\right)$ yields the strong convergence of the sequence in $H^{1}(\Omega)$.

Note that the above convergence holds for any sequence of sub-domains $\Omega_{a}^{n}$, whether it is a minimizing sequence or not. From this property, finishing the proof of the proposition follows the classical approach (see Allaire (2002) for details). In particular, by continuity, the values of the objective function $\left(\mathcal{J}\left(u_{0}\left(K\left(\Omega_{a}^{n}\right)\right)\right)\right.$ are also converging to the limit value $\mathcal{J}\left(u_{0}(K(\theta))\right.$. As a consequence, the infimum of equation (27) remains unchanged by replacing the variable set, $\left\{K\left(\Omega_{a}\right) \mid \Omega_{a} \subset \Omega\right\}$, with its weak closure, that is the set $\left\{K(\theta) \mid \theta \in L^{2}(\Omega,[0,1])\right\}$ (see Lemma 4.2 in $\operatorname{Tartar}(2009))$.

The term minimizing sequences refers to sequences of material distributions whose cost function values are decreasing to the infimum. The limit coefficient, $K(\theta)$ is thus in general not of the form $K\left(\Omega_{a}^{*}\right)$, for a certain sub-domain $\Omega_{a}^{*} \subset \Omega$, since the limit density $\theta$ can take any pointwise values between 0 and 1 .

This relaxation result (equation 30 has an important consequence. Numerically it is observed that a minimizing sequence of bi-material wear coefficients, 
$K\left(\Omega_{a}^{n}\right)$, converging weakly to $K(\theta)$, is in fact converging to a highly-distributed composite material with a volume fraction, $\theta(x)$ of material $A$ and $(1-\theta(x))$ of material $B$, at every point, $x \in \Omega$.

When a material discontinuity is present in the second order differential operator of the elliptic equation (for example when considering discontinuous thermal conductivity or elasticity tensors), the weak convergence of the material distribution does not imply the strong convergence of the associated solution. Indeed, it does not imply that the convergence of the differential operator depends on the material distribution. Instead, homogenization theory predicts that additional sub-sequences must be extracted to obtain the convergence (in the sense of $H$-convergence) of the differential operator to an effective, homogenized, tensor. In the context of periodic homogenization theory (Murat and Tartar (1985); Allaire (2002); Bendsøe and Kikuchi (1988)), the properties of the homogenized tensor are characterized by a unit-cell problem that defines a microstructure for the limit anisotropic material. The infimum of the objective function is attained for both a limit fractional distribution of the two materials, and an asymptotic anisotropy described by the homogenized limit of the differential operator. In the case of the steady-state equation (24), the differential operator does not depend on the material distribution and thus does not lead to effective anisotropic homogenized tensors.

Thus, as is highlighted by the relaxation result of equation 30 , the limit volume fraction is the sole parameter driving the optimization process. Within the field of tribology, it has been experimentally established that wear properties of composite materials do in fact depend on their physical microstructures or anisotropy (Zmitrowicz (2006); Sawyer et al (2014)). This highlights the limitations of the underlying wear model: it is limited at small scales when the distribution of materials within the composite would begin to affect the microstructure of its constituents. This is not surprising because Archard's law (equation 1) is itself based on the empirical observation that the average worn volume of material is proportional to the sliding distance. If the microstructure of the constituent materials is significantly altered, the assigned wear rates would no longer be valid. Therefore the law breaks down at a scale that is comparable to the size of the fragments of worn material (Zum Gahr (1987)).

Nevertheless this model remains suitable for optimizing the wear performance of multimaterial composites as long as a physical length scale for a minimum feature size is specified. For example, a minimum thickness length scale in each material phase or a minimum radius of curvature along material interfaces may be prescribed. Numerically, identifying microstructures with target wear performance and desired feature control is the focus of the following Section 5 Topology optimization will be used to improve upon typical composite wear designs without significant increase in geometric complexity.

4 Defining optimization objectives: minimizing run-in-wear volume and achieving planar steady-state surfaces with multimaterial composites

4.1 Minimizing run-in-wear volume loss

Improving the wear performance of tribological systems can be done by minimizing the volume of material lost during the transient run-in period (here equal to 
$\left.-\int_{\Omega} z(s, x) \mathrm{d} x\right)$. By definition, wear involves material removal, but material lost during transient wear is considered a waste to be minimized. This material loss quantity is defined with respect to a given sliding distance, $s$, and is consequently time-dependent. Instead, equation (13) simplifies the analysis by providing an asymptotic expansion for the volume lost:

$$
-\int_{\Omega} z(s, x) \mathrm{d} x=P_{0}<K^{-1}>^{-1}|\Omega| s-\left(\int_{\Omega} \frac{<K^{-1}>^{-1}}{K} z_{0} \mathrm{~d} x+\int_{\Omega} u \mathrm{~d} x\right)+G(s),
$$

where $G(s)$ is decreasing exponentially to zero with the sliding distance, $s$.

Equation (31) shows that the first term to be considered when comparing the volume of material lost between two material distributions, is the quantity: $P_{0}<K^{-1}>^{-1}|\Omega| s$. Consequently, to first order, the volume of material lost is related to the equivalent wear rate, $\left\langle K^{-1}\right\rangle^{-1}$, and the asymptotic comparison of two initial distributions has a physical meaning only if their respective equivalent wear rates are equal. As the equivalent wear rate

$$
<K^{-1}>^{-1}=\left[\left(\frac{1}{K_{a}}-\frac{1}{K_{b}}\right) \frac{\left|\Omega_{a}\right|}{|\Omega|}+\frac{1}{K_{b}}\right]^{-1}
$$

depends only on the proportion of the domain, $\Omega_{a}$ filled by material $A$, this constraint implies that the volume fractions $\left(\theta^{0}\right.$ and $1-\theta^{0}$ for the constituent materials $A$ and $B$ ) must be fixed in the domain:

$$
\left|\Omega_{a}\right|=\theta^{0}|\Omega| .
$$

Once the volume fraction, $\theta^{0}$, is prescribed, the comparison between two configurations of materials can be made by examining the second term of the asymptotic expansion (31):

$$
V_{\text {lost }}=-\left(\int_{\Omega} \frac{<K^{-1}>^{-1}}{K} z_{0} \mathrm{~d} x+\int_{\Omega} u \mathrm{~d} x\right)=\int_{\Omega} \frac{<K^{-1}>^{-1}}{K}\left(u_{0}-z_{0}\right) \mathrm{d} x .
$$

This quantity is the volume of material lost due to the transient run-in wear period when the initial profile, $z_{0}$, converges to the steady-state profile, $u_{0}$. In this way, the quantity $V_{\text {lost }}$, defined by (33), enables one to compare the wear performance of material distributions for any choice of initial profile, $z_{0}$. In the case of an initially flat profile, $z_{0}=0$, the more general quantity, $V_{\text {lost }}$, is called the runin-wear volume, $V_{\text {run-in }}$, in deference to the terminology used by the tribology community (Sawyer et al (2014); Chattopadhyay (2001)):

$$
V_{\text {run-in }}=J(K)\left(u_{0}\right)=\int_{\Omega} \frac{<K^{-1}>^{-1}}{K} u_{0} \mathrm{~d} x .
$$

The run-in-wear volume is determined assuming a known in-plane material distribution, $K$, and a fixed value of the equivalent wear rate, $\left\langle K^{-1}>^{-1}\right.$. This unambiguously defines what is usually interpreted by tribologists as the volume lost before reaching steady-state wear (Hirst and Lancaster $(1956))$. The optimization problem is then set on the fixed space, $V_{1}=\left\{v \in H^{1}(\Omega) \mid<v>=0\right\}$ : 


$$
\inf _{\Omega_{a} \subset \Omega} J(K)\left(u_{0}\right)
$$

under the constraints:

$$
\left\{\begin{aligned}
K & =K\left(\Omega_{a}\right)=\left(K_{a}-K_{b}\right) \mathbf{1}_{\Omega_{a}}+K_{b} \\
\left|\Omega_{a}\right| & =\theta^{0}|\Omega| \\
u_{0} \in V_{1} & \\
\forall v \in V_{1}, a\left(u_{0}, v\right) & =l(v)+P_{0} J(K)(v) .
\end{aligned}\right.
$$

Note that it is not immediately obvious from the definition of equation (34), that the objective function, $J(K)\left(u_{0}\right)$, is of the type $\mathcal{J}\left(u_{0}\left(K\left(\Omega_{a}\right)\right)\right)$, as in Section 3 . Based on equation (34), $J(K)\left(u_{0}\right)$ depends not only on $u_{0}\left(K\left(\Omega_{a}\right)\right)$, but also on $K\left(\Omega_{a}\right)$. However $J(K)\left(u_{0}\right)$ is indeed of the type $\mathcal{J}\left(u_{0}\left(K\left(\Omega_{a}\right)\right)\right)$, because the following equality is found (equation (35)):

$$
J(K)\left(u_{0}\right)=\frac{1}{P_{0}}\left(a\left(u_{0}, u_{0}\right)-l\left(u_{0}\right)\right) .
$$

The relaxation result of Proposition 3 holds true in this case.

The shape derivative with respect to the interface, $\partial \Omega_{a}$, is obtained using Céa's fast derivation method (Céa (1986)). The following Lagrangian is defined for any $\hat{u}_{0}, \hat{p} \in V_{1}$ and $\Omega_{a} \subset \Omega$ :

$$
\mathcal{L}\left(\hat{u}_{0}, \hat{p}, \Omega_{a}\right)=J(K)\left(\hat{u}_{0}\right)-a\left(\hat{u}_{0}, \hat{p}\right)+l(\hat{p})+P_{0} J(K)(\hat{p}) .
$$

The notation^ in equation (37) means that the functions $\hat{u}_{0}, \hat{p}$ are not the solutions

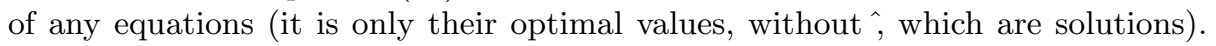
The adjoint-state, $p$ is defined as the unique solution in $V_{1}$ of:

$$
\forall v \in V_{1}, \quad \frac{\partial \mathcal{L}}{\partial u_{0}} \cdot v=J(K)(v)-a\left(v, p_{0}\right)=0,
$$

where $\frac{\partial \mathcal{L}}{\partial u_{0}} \cdot v$ denotes the directional partial derivative of the Lagrangian with respect to $u_{0}$ in the direction $v$. In the physical case where $a$ and $l$ are given by equations (8) and (9), $p$, is defined as the solution of:

$$
\left\{\begin{array}{rlrl}
k_{s}(p-<p>)-k_{g}(\Delta p-<\Delta p>) & =-1+\frac{<K^{-1}>^{-1}}{K} & \text { in } \Omega \\
k_{g} \frac{\partial p}{\partial n}+\beta(p-<p>) & =0 & & \text { on } \partial \Omega \\
<p> & =0 . &
\end{array}\right.
$$

Following the classical approach (Céa (1986); Allaire et al (2004)), the shape derivative of the objective function is given by $\frac{\mathrm{d} J}{\mathrm{~d} \Omega_{a}} \cdot \boldsymbol{\theta}=\frac{\partial \mathcal{L}}{\partial \Omega_{a}}\left(u_{0}, p, \Omega_{a}\right) \cdot \boldsymbol{\theta}$, where the Lagrangian is evaluated in $u_{0}$, satisfying the constraints of equation (35) and 
in $p$, the adjoint. Then a standard computation yields the shape derivative of the objective function:

$$
\begin{aligned}
& \frac{\mathrm{d} J}{\mathrm{~d} \Omega_{a}} \cdot \boldsymbol{\theta}= \frac{\partial}{\partial \Omega_{a}}\left(J(K)\left(u_{0}+P_{0} p\right)\right) \cdot \boldsymbol{\theta} \\
&=<K^{-1}>^{-1}\left(\frac{1}{K_{a}}-\frac{1}{K_{b}}\right) \int_{\partial \Omega_{a}}\left(u_{0}+P_{0} p\right. \\
&\left.\quad \quad\left\langle\frac{\left\langle K^{-1}>^{-1}\right.}{K}\left(u_{0}+P_{0} p\right)\right\rangle\right) \boldsymbol{\theta} \cdot \mathbf{n d} s .
\end{aligned}
$$

In practice, the resolution of the system (39) for the adjoint state can be performed from Proposition 2. The equality volume constraint, $\left|\Omega_{a}\right|=\theta^{0}|\Omega|$, will be addressed in Section 5.

\subsection{Achieving a planar steady-state profile}

An alternative wear optimization objective may be a desired surface roughness or topography. The following subsection focuses on the case when a planar steadystate profile with no roughness at all must be achieved. This requires a new objective function and it is shown next that compliance is a good candidate. By analogy with linear elasticity, one can define compliance in this wear model to be the work done by the loads :

$$
l(u)=-\int_{\Omega} f u \mathrm{~d} x+\int_{\partial \Omega} g(u-<u>) \mathrm{d} s .
$$

Minimizing compliance is a very common objective or cost function in structural optimization for linear elastic problems. However the problem considered is not strictly equivalent, namely the linear forcing, $l$, depends on the material distribution, $K$. By virtue of equation (11) compliance satisfies $l(u)=a(u, u)$, and further examination of equation (8) reveals that $a(u, u)$ is a measure of how far the profile, $u$, is from being a constant. Thus compliance can also be interpreted as the cost function associated with the objective of achieving a flat steady-state profile. Noting that $a(u, u)=a\left(u_{0}, u_{0}\right)$, the optimization problem is defined on the space $V_{1}$ by:

$$
\inf _{\Omega_{a} \subset \Omega} \mathcal{J}\left(u_{0}\left(K\left(\Omega_{a}\right)\right)\right)=a\left(u_{0}, u_{0}\right),
$$

under the same constraints 35 . The shape derivative is calculated by introducing the Lagrangian:

$$
\mathcal{L}\left(\hat{u}_{0}, \hat{p}, \Omega_{a}\right)=a\left(\hat{u}_{0}, \hat{u}_{0}\right)-a\left(\hat{u}_{0}, \hat{p}\right)+l(\hat{p})+P_{0} J(K)(\hat{p}) .
$$

The adjoint-state, $p$, is now defined as:

$$
\forall v \in V_{1}, \quad \frac{\partial \mathcal{L}}{\partial u_{0}} \cdot v=2 a\left(v, u_{0}\right)-a(v, p)=0 .
$$

It is deduced from equation (43) that $p=2 u_{0}$, and the problem is self-adjoint. The shape derivative is $\frac{\mathrm{d} \mathcal{J}}{\mathrm{d} \Omega_{a}} \cdot \boldsymbol{\theta}=\frac{\partial}{\partial \Omega_{a}}\left(J(K)\left(2 u_{0}\right)\right) \cdot \boldsymbol{\theta}$, and a computation yields: 


$$
\frac{\mathrm{d} \mathcal{J}}{\mathrm{d} \Omega_{a}} \cdot \boldsymbol{\theta}=2<K^{-1}>^{-1}\left(\frac{1}{K_{a}}-\frac{1}{K_{b}}\right) \int_{\partial \Omega_{a}}\left(u_{0}-\left\langle\frac{\left\langle K^{-1}>^{-1}\right.}{K} u_{0}\right\rangle\right) \boldsymbol{\theta} \cdot \mathbf{n} \mathrm{d} s
$$

5 A topologically sensitive optimization algorithm with equality volume constraints and perimeter penalization

\subsection{Topology optimization of run-in wear with periodic boundary conditions}

In the following, the focus is on optimizing the unit-cell of a bi-material composite by minimizing the associated run-in wear volume (34). The unit-cell is a rectangular domain $\Omega=[0, L] \times[0, H]$ and periodic boundary conditions are assumed. In this case, the reference pressure profile is a constant, $f=P_{0}$. As stated above, the previous results from Section 4 obtained for homogeneous Neumann boundary conditions $(g=\beta=0)$ can be applied, without modification, to the case of periodic boundary conditions.

With periodic boundary conditions and a constant pressure profile, minimizing the run-in-wear volume (Section 4.1) is identical to minimizing compliance (Section 4.2). This is because both objective functions, $J(K)\left(u_{0}\right)$, defined by (34), and $l(u)$, defined by (42), are positively proportional (using equations 20 and (23):

$$
l(u)=-P_{0} \int_{\Omega} u \mathrm{~d} x=P_{0} J(K)\left(u_{0}\right) .
$$

The global minimum of the objective function, $J(K)\left(u_{0}\right)$, under the constraints (35) is zero; this is because compliance can also be interpreted as an energy: $P_{0} J(K)\left(u_{0}\right)=a\left(u_{0}, u_{0}\right) \geqslant 0$. In view of relation 200 , the minimum of the relaxed formulation $(29)$ is attained by constant profiles, $u_{0}$, or equivalently, by uniform material distributions, $K=\left\langle K^{-1}>^{-1}\right.$, corresponding to vanishing loads 22 for $u_{0}$. As a result of Proposition 3 any minimizing sequence $K\left(\Omega_{a}^{n}\right)$ of the original problem 27) has the property that its inverse is weakly converging to a constant and reciprocally, any sequence that converges weakly to a constant is on a minimizing trajectory. Numerically, the minimizing sequences, $K\left(\Omega_{a}^{n}\right)$, exhibit features that become vanishingly small while still respecting the required fixed volume fractions of materials at every point in the domain. Figure 6 presents a typical
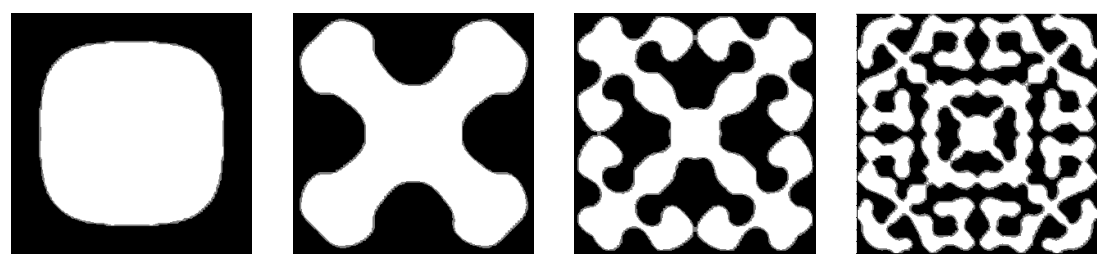

Fig. 6: A minimizing sequence of a bi-material distribution converging weakly to a constant, while respecting a resource constraint ratio of $50 \%$ for each material at each iteration. 
example of minimizing sequences obtained using a level-set based topology optimization algorithm that imposes an equality volume constraint at each iteration (see Section 5.3). Proceeding left to right, the microstructural features increase in complexity and a highly-interconnected distribution of materials is observed. These minimizing sequences are converging to the theoretical limit in which every point in the domain is occupied by the prescribed volume fraction of materials. For example, if a resource constraint with a volume fraction of $50 \%$ material A (white) and $50 \%$ material B (black) was specified, the optimal solution would be a domain in which every material point is comprised of $50 \%$ material A and $50 \%$ material B (the domain would look completely grey).

For practical wear applications, it is desirable to avoid these types of densitylike solutions with finely distributed materials. The reasons are those mentioned in Section 3 as well as considerations of manufacturability. Manufacturing and fabrication considerations limit the complexity of conceivable designs. This is a challenge commonly encountered in topology optimization (Allaire et al (2014b); Guest et al $(2004)$; Meisel et al (2013); Jansen et al (2013); Zhou et al (2014); Alexandersen and Lazarov (2015); Schury et al (2012)) - geometric constraints on the boundary, $\partial \Omega_{a}$, must be added to ensure that minimizing sequences of the domain converge toward a manufacturable shape (Delfour and Zolésio (2001); Ambrosio and Buttazzo (1993)). Several methods are now available to address manufacturability: these include perimeter penalization, minimum thickness constraints, molding constraints, and robust topology optimization (Allaire et al $(2014 \mathrm{~b})$; Guest et al (2004); Zhou et al (2014); Alexandersen and Lazarov (2015); Wang et al (2011); Schevenels et al (2011); Sigmund (2009); Seepersad et al (2006)).

Methods that rely on the shape derivative of the signed distance function can be difficult to handle numerically because they may require integration along rays normal to the interface (Allaire et al (2014b a )). Projection methods that optimize the density of material often require extra penalizations to avoid graded materials (Jansen et al (2013)). For these reasons, in the context of level-set methods, perimeter penalization remains a convenient way to control the geometric complexity of optimized structures. Although there is no formal guarantee that the perimeter constraint will translate into manufacturable standards, it often guarantees the existence of optimal shapes - hence convergence -, that are expected to be simplified, improving the chance of manufacturability in a general way (not specific to a manufacturing process). In level-set methods, penalization schemes are usually incorporated in the level-set transport equation through a diffusive term that can take the form of a surface energy (Chen et al (2007); Luo et al (2008); Yamada et al (2010)). The diffusive term tends to regularize the level-set. Simultaneously, the equality volume constraint on the constituent materials must be accounted for in the optimization process. To address these issues, a topology optimization method that is suitable for a broad range of applications and that takes a novel approach to implement perimeter penalization and resource constraints is presented in the following section.

\subsection{Lagrange multiplier for the equality volume constraint}

Several techniques are available to handle equality constraints in gradient-based optimization algorithms (Bertsekas (1996)). In this work, a multiplier, $\lambda$, for the 
equality volume constraint $\left(\left|\Omega_{a}\right|=\theta^{0}|\Omega|\right)$ is introduced and the following Lagrangian (different from the ones introduced in Section 44) is considered:

$$
\mathcal{L}\left(\Omega_{a}, \lambda\right)=J(K)\left(u_{0}\right)+\tau\left|\partial \Omega_{a}\right|+\lambda\left(\left|\Omega_{a}\right|-\theta^{0}|\Omega|\right) .
$$

The shape derivative of the Lagrangian is given by:

$$
\begin{aligned}
\frac{\partial \mathcal{L}}{\partial \Omega_{a}} \cdot \boldsymbol{\theta} & =\frac{\mathrm{d} \mathcal{J}}{\mathrm{d} \Omega_{a}} \cdot \boldsymbol{\theta}+\tau \int_{\partial \Omega_{a}} \kappa \boldsymbol{\theta} \cdot \mathbf{n} \mathrm{d} s+\lambda \int_{\partial \Omega_{a}} \boldsymbol{\theta} \cdot \mathbf{n} \mathrm{d} s \\
& =\int_{\partial \Omega_{a}}(V+\tau \kappa+\lambda) \boldsymbol{\theta} \cdot \mathbf{n} \mathrm{d} s
\end{aligned}
$$

where $\kappa$ is the mean curvature of the boundary, $\partial \Omega_{a}$, and $V$ is the velocity in the shape derivative (equation 44 of the cost function, $J(K)\left(u_{0}\right)$ :

$$
V=2\left\langle K^{-1}\right\rangle^{-1}\left(\frac{1}{K_{a}}-\frac{1}{K_{b}}\right)\left(u_{0}-\left\langle\frac{<K^{-1}>^{-1}}{K} u_{0}\right\rangle\right) .
$$

A descent direction for the Lagrangian is obtained by considering a vector field, $\boldsymbol{\theta}$, whose value on the interface, $\partial \Omega_{a}$, is $\boldsymbol{\theta}=-(V+\tau \kappa+\lambda) \mathbf{n}$. At each iteration, the value of the multiplier, $\lambda$, is updated to satisfy the equality volume constraint, $\left|\Omega_{a}\right|=\theta^{0}|\Omega|$.

5.3 A novel modification of the level-set based topology algorithm to impose an equality volume constraint

Several methods exist to represent and evolve numerically the interface of a domain Chen et al (2007); Dapogny (2013). The level-set method is used in this paper, for its simplicity in allowing topological changes caused by the collision of moving frontiers to occur (Osher and Sethian (1988); Allaire et al (2004); Wang et al (2003)). The domain, $\Omega$, is discretized on a fixed mesh and the distribution of materials is described by a level-set function, $\phi$, satisfying:

$$
\begin{cases}\phi>0 & \text { in } \Omega_{a} \\ \phi<0 & \text { in } \Omega \backslash \overline{\Omega_{a}} \\ \phi=0 & \text { on } \partial \Omega_{a} .\end{cases}
$$

The domain $\Omega_{a}$ is updated at each iteration by moving each point of the boundary along its outer normal at the speed $v$, using the following Hamilton-Jacobi equation Osher and Sethian (1988)):

$$
\frac{\partial \phi}{\partial t}-v|\nabla \phi|=0
$$

The negative sign in front of the speed, $v$, results from the convention that $\phi$ must be positive in $\Omega_{a}$ (see equation (48)). In order to ensure numerical stability and to guarantee the accuracy of the finite-difference schemes used to solve this equation, the level-set function, $\phi$, is usually initialized as the opposite of the signed-distance function, $d_{\Omega_{a}}$, of the domain $\Omega_{a}$ (Osher and Fedkiw (2004)). This property is maintained at each iteration by a reinitialization procedure (Osher and Fedkiw (2004)). No explicit relationship is available a priori that relates the 
multiplier, $\lambda$, and the volume, $\left|\Omega_{a}\right|$, of the updated domain. The multiplier, $\lambda$, can be determined by dichotomy (Allaire (2002)) or by augmented Lagrangian methods (Bertsekas (1996)). Using a dichotomy may be computationally costly as the domain may need to be updated several times before the correct value of the multiplier, $\lambda$, is found. A disadvantage of the augmented Lagrangian approach is that the value of the volume multiplier, $\lambda$, is correct only at convergence. The implication is that the objective function does not necessarily decrease at each iteration.

To avoid these issues, a protocol that includes a novel constraint on the level-set function is proposed instead. An approximate, but affine relationship between the Lagrange multiplier and the volume, $\left|\Omega_{a}\right|$, is proposed. Furthermore, it is shown that the expression of the topological gradient is a straightforward extension of the speed of the shape derivative inside the domain. This has the added benefit of allowing for the nucleation of a material inclusion $(A$ or $B)$.

In order to simplify the volume calculation, a small parameter, $\epsilon>0$, is introduced and a further constraint that the level-set function, $\phi$, must be constant in each material domain at a distance, $\epsilon$, from the interface, $\partial \Omega_{a}$, is imposed (Figure 7):

$$
\phi=2 H_{\epsilon}\left(-d_{\Omega_{a}}\right)-1,
$$

where $H_{\epsilon}$ is a regularized Heaviside function over an interface zone of thickness $2 \epsilon$. The regularization proposed by Osher and Fedkiw (2004) is applied:

$$
H_{\epsilon}(x)= \begin{cases}0 & \text { if } x \leqslant-\epsilon \\ \frac{1}{2}+\frac{x}{2 \epsilon}+\frac{1}{2 \pi} \sin \left(\frac{\pi x}{\epsilon}\right) & \text { if }-\epsilon \leqslant x \leqslant \epsilon \\ 1 & \text { if } x \geqslant \epsilon .\end{cases}
$$
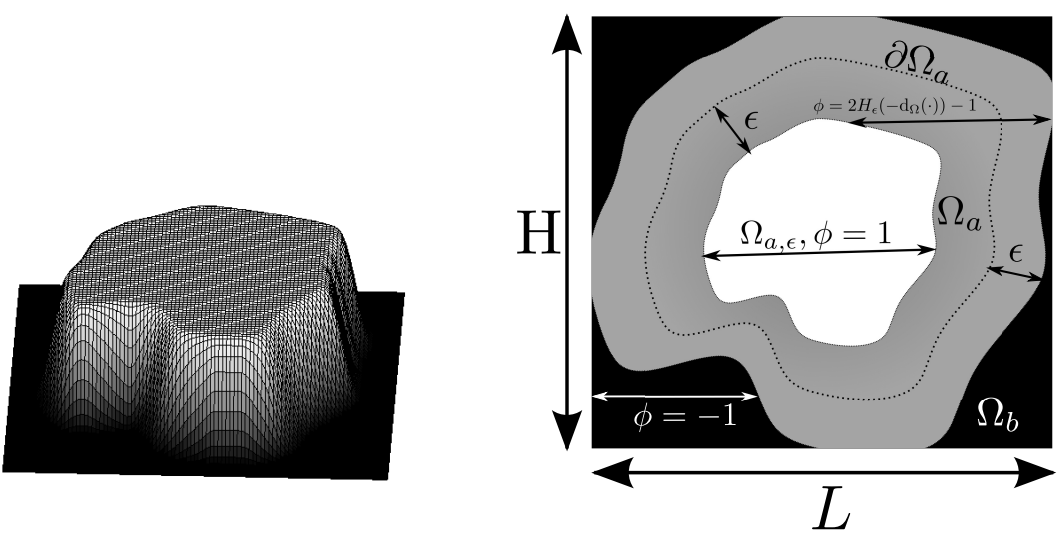

Fig. 7: On the left, is a 3D profile of the level-set function. On the right: the interpolation zone for the level-set.

Thus $\phi$ is still a level-set function for $\Omega_{a}$ because the constraint equation 48 is satisfied; but it also has a value that is constant (either -1 or +1 ) at a fixed distance, 
$\epsilon$, from the boundary (Figure 7). The practical interest of equation 50 is that the volume, $\left|\Omega_{a}\right|$, is related to the level-set, $\phi$, by means of an affine relationship. Indeed, denoting $\Omega_{a, \epsilon}=\left\{x \in D \mid d_{\Omega_{a}}(x) \leqslant-\epsilon\right\}$, and assuming the interface, $\partial \Omega_{a}$, is a closed smooth curve, an application of a coarea formula and Gauss-Bonnet theorem yields the affine relation (Chavel (2010)):

$$
\begin{aligned}
\int_{\Omega} \frac{\phi(x)+1}{2} \mathrm{~d} x & =\int_{\Omega} H_{\epsilon}\left(-d_{\Omega_{a}}(x)\right) \mathrm{d} x \\
& =\left|\Omega_{a, \epsilon}\right|+\int_{\partial \Omega_{a}} \int_{-\epsilon}^{\epsilon} H_{\epsilon}(z)(1-\kappa(y) z) \mathrm{d} y \mathrm{~d} z \\
& =\left|\Omega_{a, \epsilon}\right|+\epsilon|\Gamma|-\frac{\epsilon^{2}}{3}\left(1+\frac{3}{\pi^{2}}\right) 2 \pi N_{\partial \Omega}
\end{aligned}
$$

This is compared to the actual volume, $\left|\Omega_{a}\right|$ :

$$
\left|\Omega_{a}\right|=\left|\Omega_{a, \epsilon}\right|+\int_{\partial \Omega_{a}} \int_{-\epsilon}^{0}(1+z \kappa(y)) \mathrm{d} z \mathrm{~d} y=\left|\Omega_{a, \epsilon}\right|+\epsilon|\Gamma|-\epsilon^{2} \pi N_{\partial \Omega_{a}},
$$

where $N_{\partial \Omega_{a}}$ is the index or winding number of the curve $\partial \Omega_{a}$ (defined here in two dimensions), which is also the number of holes or inclusions in the material distribution. In this way the quantity defined by equation 52 is a good approximation for the volume, $\left|\Omega_{a}\right|$, as long as: (i) the number of inclusions, $N_{\partial \Omega_{a}}$ and the parameter $\epsilon$ remain small, and (ii) the extent of the domain, $\Omega_{a}$, is greater than $\epsilon$ (Delfour and Zolésio (2001)). This affine relationship will be exploited to determine an explicit formula for the Lagrange multiplier at each iteration. In the numerical implementation, the signed distance function is computed at each iteration from the knowledge of the level-set function $\phi$. The Heaviside function, $H_{\epsilon}$, is subsequently applied to ensure that the constraint equation 50 on the level-set is satisfied at the beginning of the next iteration.

5.4 Updating scheme for moving the boundary and allowing the nucleation of inclusions

The transport equation $49 \mathrm{p}$ is used to move the interface, $\partial \Omega_{a}$, at each iteration. At the beginning of each iteration, the level-set function, $\phi$, is defined by equation (50), which implies that its gradient and its Laplacian are given by: $\nabla \phi=-2 \overline{H_{\epsilon}^{\prime}}\left(-d_{\Omega_{a}}\right) \nabla d_{\Omega_{a}}$ and $\Delta \phi=2 H_{\epsilon}^{\prime \prime}\left(-d_{\Omega_{a}}\right)-2 H_{\epsilon}^{\prime}\left(-d_{\Omega_{a}}\right) \Delta d_{\Omega_{a}}$, respectively. In particular, the following equalities are obtained at the interface, $\partial \Omega_{a}$ :

$$
\nabla \phi=-\frac{2}{\epsilon} \mathbf{n} \quad \text { and } \quad \Delta \phi=-\frac{2}{\epsilon} \kappa
$$

Then, assuming that these relations remain approximately exact during the next iteration, the transport equation 49 with the velocity, $v=-(V+\tau \kappa+\lambda)$, can be written at the interface, $\partial \Omega_{a}$ :

$$
\frac{\partial \phi}{\partial t}-\tau \Delta \phi=-\frac{2}{\epsilon}(V+\lambda)
$$


This equation (extended to the whole domain, $\Omega$ ) for updating the level-set function, $\phi$, is convenient because it is affine with respect to $\phi$. With the modified transport equation (54), perimeter penalization is achieved by the diffusive term $(-\tau \Delta \phi)$, which regularizes the level-set function (Allaire (2007b); Yamada et al (2010)). As remarked in Osher and Fedkiw (2004), the level-set function solving equation (54) does not respect the constraint (50) for $t>0$. Thus equation (54) is just a convenient approximation of the advection equation (49), under the constraint $(50)$, over a sufficiently small time interval $[0 ; \mathrm{d} t]$. This approximation would be exact for a velocity field, $v$, constant along the normal vector, $\mathbf{n}$, and if no topological change occurs (see section 3 in Allaire et al (2014a) and Gomes and Faugeras (1999)). After a short time, d $t$, the level-set function must be reinitialized by recomputing it through equation 50 .

Yamada et al (2010) used the same equation (54) and interpreted it as a gradient descent scheme for optimizing a criterion formulated in terms of the level-set function, $\phi$. The main differences in Yamada et al (2010) compared to the approach presented here are, first, in the way equation (54) is introduced and motivated, and second, in the fact that the topological derivative was used instead of the shape gradient. As a consequence, reinitializing the levelset function was not possible as it would affect the value of the objective function. Furthermore, the width of the interpolation zone was not controlled, leading to potential numerical issues (creating an artificial interpolation zone may decrease the cost function). In contrast to Yamada et al (2010), equation (54) is interpreted here as a transport equation whose single purpose is to update the geometry of the domain, $\Omega_{a}$. To comply with the constraint (50), the level-set function is regularly reinitialized without interfering with the cost function.

The perimeter penalization term $(|\partial \Omega|)$ is evaluated in practice by using equation 50 , which yields $\nabla \phi=-2 H_{\epsilon}^{\prime}\left(-d_{\Omega_{a}}\right) \nabla d_{\Omega_{a}}$, and a coarea formula:

$$
|\partial \Omega|=\frac{\epsilon}{3} \int_{\Omega}|\nabla \phi|^{2} \mathrm{~d} x
$$

This is convenient because computing $\int_{\Omega}|\nabla \phi|^{2} \mathrm{~d} x$ is straightforward using the associated finite element stiffness matrix. Again, one can note that the fictitious energy introduced in Yamada et al (2010) to penalize the moving interface is found but, in the present approach, it is rigorously related to the perimeter by equation (55).

So far, equation (54) is justified only on the boundary $\partial \Omega_{a}$, although it is used in numerical practice, throughout the whole domain $\Omega$. A justification is now given such that equation (54) may be used in the whole domain without modification. Indeed, the velocity, $V$, defined by equation (47), is valid, not only on $\partial \Omega_{a}$, but on the whole domain, because its expression is identical to the topological gradient of the objective function, $J(K)\left(u_{0}\right)$ (Céa et al (2000); Sokolowski and ' Zochowski $(1999))$. The topological gradient, $\mathrm{d}_{\mathcal{T}} \mathcal{J}\left(x_{0}\right)$, of a cost function, $\mathcal{J}$, at a point, $x_{0} \in \Omega$, is defined when the following asymptotic expansion holds true:

$$
\mathcal{J}\left(\Omega_{a} \cup \mathcal{B}\left(x_{0}, \rho\right)\right)=\mathcal{J}\left(\Omega_{a}\right)+\rho^{d}\left|\omega_{d}\right| \mathrm{d}_{\mathcal{T}} \mathcal{J}\left(x_{0}\right)+o\left(\rho^{d}\right),
$$

where $\mathcal{B}\left(x_{0}, \rho\right)$ is the ball of center $x_{0}$ and radius $\rho$; and $d$ is the dimension of the domain and $\left|\omega_{d}\right|$ is the measure of the unit ball in dimension $d$. As a result, 
when $\mathrm{d}_{\mathcal{T}} \mathcal{J}$ is negative, it is advantageous to nucleate a small inclusion filled with material $A$ at $x_{0}$. Since the level-set function has a constant value, $\phi \in\{-1,1\}$, at a distance larger than $\epsilon$ from the interface $\partial \Omega_{a}$, following the approach of He et al (2007); Yamada et al (2010); Allaire and Jouve (2006), a gradient descent on the level-set function (here normalized by the coefficient $\frac{2}{\epsilon}$ ) can be used to nucleate an inclusion:

$$
\frac{\partial \phi}{\partial t}=-\frac{2}{\epsilon} \mathrm{d}_{\mathcal{T}} \mathcal{J}
$$

This scheme is expected to nucleate holes or inclusions wherever the topological derivative, $\mathrm{d}_{\mathcal{T}} \mathcal{J}$, is sufficiently large. The determination of how large is large enough is made by comparing the value of the topological derivative to the coefficient, $\frac{\epsilon}{2 \mathrm{~d} t}$, where $\mathrm{d} t$ is the time increment used in the discretization of equation (57). It should be noted that the perimeter is not topologically differentiable according to the definition given by equation (56); as such it is not taken into account in the optimization scheme (57). Any decrease in the objective function gained by adding new inclusions should outweigh the associated costs incurred by increasing the perimeter. It is indeed shown in the next section that an appropriate discretization of the scheme (54) allows one to prevent the topological derivative from creating too many holes or inclusions and impacting the penalization $\tau\left|\partial \Omega_{a}\right|$. As the level-set function is constant (equal to \pm 1 ) at a distance, $\epsilon$, from the interface, $\partial \Omega_{a}$, and its Laplacian is zero $(\Delta \phi=0)$, the implication is that the equations (54) and (57), moving the interface and updating the topology, are identical at a distance, $\epsilon$, from the boundary, $\partial \Omega_{a}$.

In this case, one can show using the considerations of Section 3 that the extension of the speed, $V$, defined in equation (47) is equivalent to the expression of the topological derivative of the objective function, $J(K)\left(u_{0}\right)$. It can thus be used as a descent direction for the objective function with respect to the topology away from the interface, $\partial \Omega_{a}$. In this way, equation (54) is used on the whole domain, $\Omega$, to update the topology and the geometry of the interface, $\partial \Omega_{a}$.

It should be noted that the expression for the topological derivative is, in general, different from the one for the shape derivative (see Allaire et al (2005)). When this is the case, the method described above can still be applied by finding a speed, $V$, for the scheme (54) that is equal to the shape derivative on the boundary, $\partial \Omega_{a}$, and to the topological gradient in $\Omega_{a, \epsilon}$ with some interpolation zone, of width $2 \epsilon$, between the two derivatives.

5.5 Discretizing scheme and updating the volume Lagrange multiplier

Equation (54) can be discretized using an implicit scheme, for example:

$$
\frac{\phi^{n+1}-\phi^{n}}{\mathrm{~d} t}-\tau \Delta \phi^{n+1}=-\frac{2}{\epsilon}(V+\lambda),
$$

where $\Delta \phi^{n+1}$ is the discretized Laplacian of the level-set function $\phi^{n+1}$, at timestep $n+1$. The speed, $V$, is also evaluated at iteration $n$. The use of an implicit scheme is numerically advantageous because it removes the need for stabilizing CFL conditions (Allaire (2007b)). This equation can be rewritten: 


$$
\phi^{n+1}=R^{-1} \phi^{n}-\frac{2}{\epsilon} \mathrm{d} t R^{-1}(V+\lambda),
$$

where $R^{-1}$ is the iteration matrix of the implicit scheme for the regularization operator $(\operatorname{Id}-\tau \mathrm{d} t \Delta)^{-1}$. As a consequence, a hole or inclusion can be nucleated only if:

$$
\left|R^{-1}(V+\lambda)\right|>\frac{\epsilon}{2 \mathrm{~d} t}\left|R^{-1} \phi^{n}\right|
$$

That is, nucleation occurs only when the regularized topological derivative $\left(R^{-1}(V+\right.$ $\lambda)$ ) is sufficiently large in comparison to the regularized level-set, $R^{-1} \phi^{n}$. The regularization naturally removes any features that are too small, suppressing the nucleation of too many holes or inclusions. For a value of the penalization parameter, $\tau$, that is sufficiently small, the amplitude of variations in the regularized level-set, $R^{-1} \phi^{n}$, and of the regularized topological gradient, $R^{-1}(V+\lambda)$, are then limited. Thus, inclusions will typically be nucleated when the value of the topological gradient is greater than $\frac{\epsilon}{2 \mathrm{~d} t}$.

Finally, an approximation is used to update the volume Lagrange multiplier, $\lambda$. A discretized version of equation (58) (either by finite differences or by finite elements) is the following linear system:

$$
S \phi^{n+1}=P \phi^{n}+Q(V+\lambda \mathbf{1}),
$$

where $P$ and $Q$ are two mass matrices, $S$ is a weighted sum of the mass and rigidity matrix that comes from the finite element discretization, and $\mathbf{1}$ is the discretized unit constant function. Although $\phi^{n+1}$ is no longer exactly given by relation 50 , one can still expect (for not too large $\mathrm{d} t$ ) that the discretization of the quantity, $\int_{\Omega} \frac{\phi^{n+1}+1}{2} \mathrm{~d} x$, is a valid approximation of the volume, $\Omega_{a}$, for the next iteration. With this assumption, the advantage of the proposed method is that one can find an approximate value of the volume Lagrange multiplier, $\lambda$, ensuring that the equality volume constraint is satisfied at the next iteration. Indeed, for the desired constraint value, $\left|\Omega_{a}\right|=\theta^{0}|\Omega|$, the finite difference scheme of equation $(58)$ can be explicitly inverted, yielding:

$$
\lambda=\frac{2 \theta^{0}-<S^{-1} P \phi^{n}+S^{-1} Q V+\mathbf{1}>}{<S^{-1} Q \mathbf{1}>},
$$

where $<\cdot>$ denotes the discretized average operator. Thus, an algorithm that handles equality volume constraints, perimeter penalization, and topological sensitivity through the use of a single updating equation is obtained.

\section{Topology optimization algorithm :}

1. For a given domain $\Omega$, solve the steady-state equation 20 and compute the shape derivative, $V$, defined by (47).

2. Compute the volume multiplier $\lambda$, using formula 62 .

3. Update the sub-domain $\Omega_{a}$ by solving equation (58).

4. Reinitialize the level-set function $\phi^{n+1}$ as follows. (i) Compute the signed-distance function of the domain $\Omega_{a}^{n+1}$, corresponding to $\phi^{n+1}$. (ii) Apply formula 50 to get the reinitialized value of $\phi^{n+1}$. 


\section{Numerical results \& discussion}

In this section, the algorithm (proposed in Section 50 is applied to optimize the unit-cell of a periodic composite material by minimizing its run-in-wear volume loss. The constituent material wear rates are taken to be $K_{a}=2.5 \times 10^{-2} \mathrm{~mm}^{3} / \mathrm{Nm}$ and $K_{b}=2.5 \times 10^{-3} \mathrm{~mm}^{3} / \mathrm{Nm}$. Materials $\mathrm{A}$ and $\mathrm{B}$ are represented in black and white, respectively. The unit-cell, $\Omega$, is square, of size $10 \mathrm{~mm} \times 10 \mathrm{~mm}$, and is discretized into a $100 \times 100$ node grid with $d x=0.1 \mathrm{~mm}$. The reference pressure is set to $f=P_{0}=0.083 \mathrm{~N} / \mathrm{mm}^{2}$, and the elastic foundation parameters (Section 2 ) are $k_{s}=0.28 \mathrm{~N} / \mathrm{mm}^{3}, k_{g}=2.8 \mathrm{~N} / \mathrm{mm}$. A resource ratio of $\theta^{0}=50 \%$ for each material is specified as the equality volume constraint. Lastly, the following parameters were set: $\epsilon=6 \mathrm{~d} x$ and $\mathrm{d} t=0.05$. At each iteration, the speed $(V+\lambda+\tau \kappa)$ was multiplied by the coefficient $\frac{\mathrm{d} t}{2 c \epsilon|\max V|}$, where $c$ is a constant set to control the effects of the topological gradient (equation 56 ).

When it is desirable to suppress the nucleation of holes or inclusions, the topological sensitivity of the algorithm can be disabled by multiplying the right-hand side of the evolution equation (54) by the regularized Dirac function, $\delta_{\epsilon}=H_{\epsilon}^{\prime}$. Since the support of $\delta_{\epsilon}$ is localized near the interface, new holes cannot be nucleated away from the boundary, $\partial \Omega_{a}$, thus cancelling the effects of the topological gradient descent (equation 57). An affine relationship remains between the volume Lagrange multiplier, $\lambda$, and the volume of $\Omega_{a}$. With this relation intact, one can conveniently impose the volume constraint with the explicit equation $\sqrt{62}$ for the Lagrange multiplier, $\lambda$. 

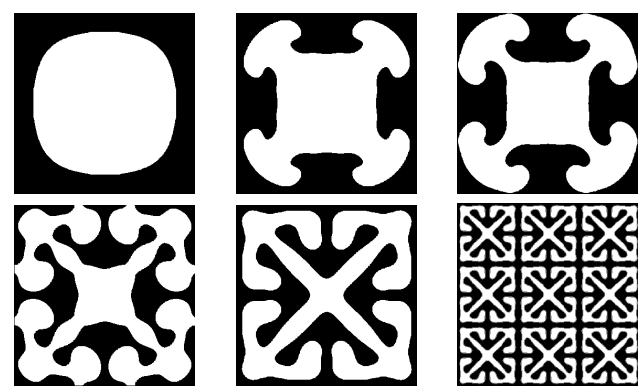

(a) Results with perimeter penalization parameter, $\tau=1.5 \times 10^{-4}$. Initial profile, Iteration 7 , 10, 17, Final design (Iteration 48), and a $3 \times 3$ tiling of the final design for periodic visualization.
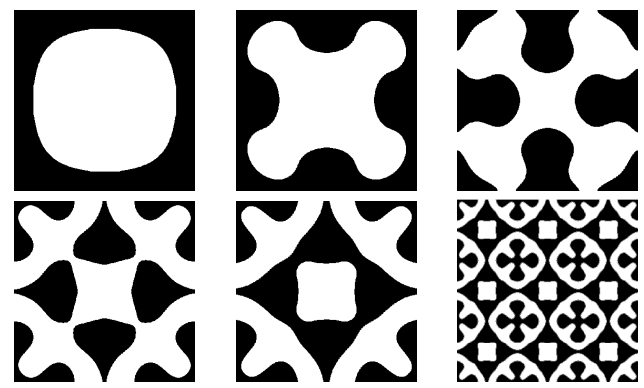

(b) Results with perimeter penalization parameter, $\tau=5 \times 10^{-3}$. Initial profile, Iteration 7,15 , 66, Final design (Iteration 84), and a $3 \times 3$ tiling of the final design for periodic visualization.
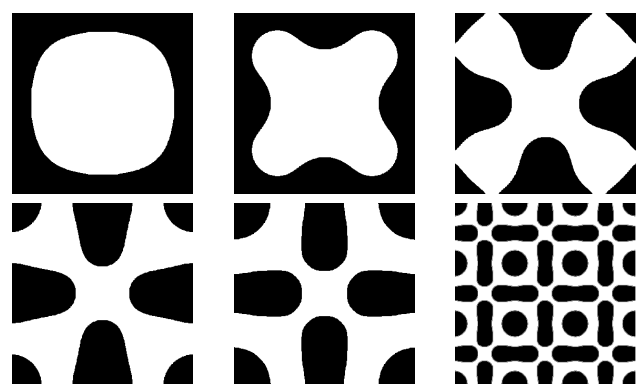

(c) Results with perimeter penalization parameter, $\tau=1.5 \times 10^{-2}$. Initial profile, Iteration 7 , 15,24 , Final design (Iteration 71 ), and a $3 \times 3$ tiling of the final design for periodic visualization.

Fig. 8: Minimizing sequences of bi-material distributions that reduce the run-in wear volume under periodic boundary conditions. Results for three values of the perimeter penalization constant, $\tau$, are shown. From left to right: the initial design, three intermediate profiles, the optimal design obtained at convergence and an extension by periodicity $(3 \times 3$ unit cells) for ease of visualization. The topological gradient is disabled. 


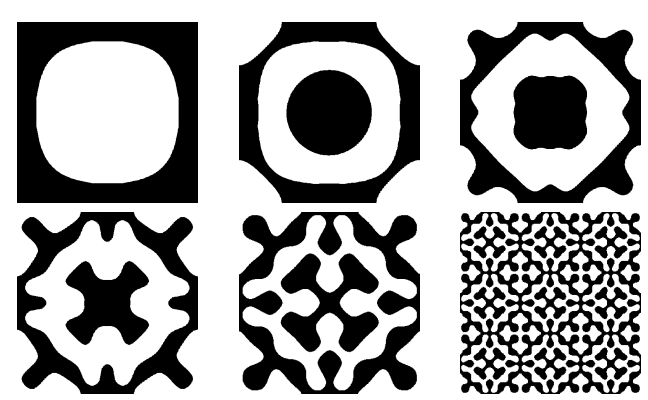

(a) Results with perimeter penalization parameter, $\tau=1.5 \times 10^{-4}$. Initial profile, Iteration 1, 25, 40, Final design (Iteration 100), and a $3 \times 3$ tiling of the final design for periodic visualization.

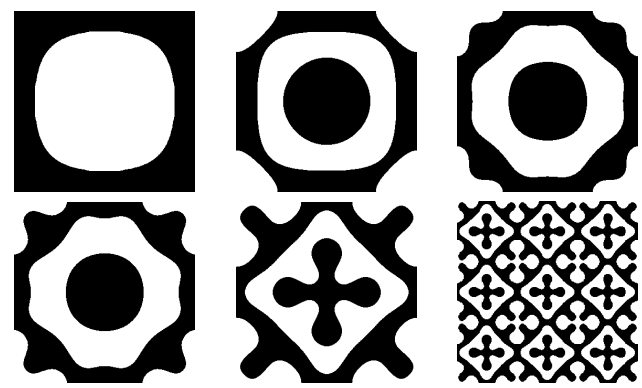

(b) Results with perimeter penalization parameter, $\tau=5 \times 10^{-3}$. Initial profile, Iteration 1,25 , 40, Final design (Iteration 100), and a $3 \times 3$ tiling of the final design for periodic visualization.
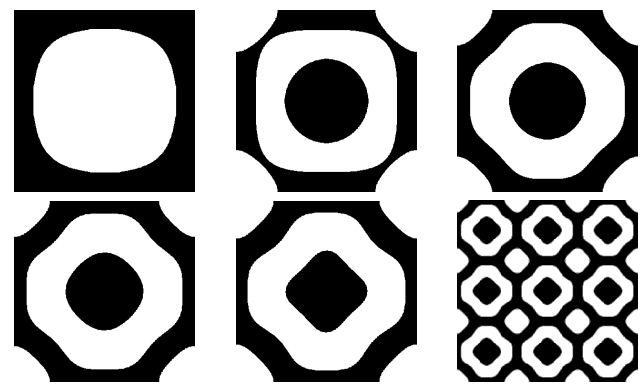

(c) Results with perimeter penalization parameter, $\tau=1.5 \times 10^{-2}$. Initial profile, Iteration 1, 25, 40, Final design (Iteration 100), and a $3 \times 3$ tiling of the final design for periodic visualization.

Fig. 9: Minimizing sequences of bi-material distributions that reduce the run-in wear volume loss under periodic boundary conditions. Results for three values of the perimeter penalization constant, $\tau$, are shown. From left to right: the initial design, three intermediate profiles, the optimal design obtained at convergence and an extension by periodicity $(3 \times 3$ unit cells $)$ for ease of visualization. The topological gradient is enabled. 

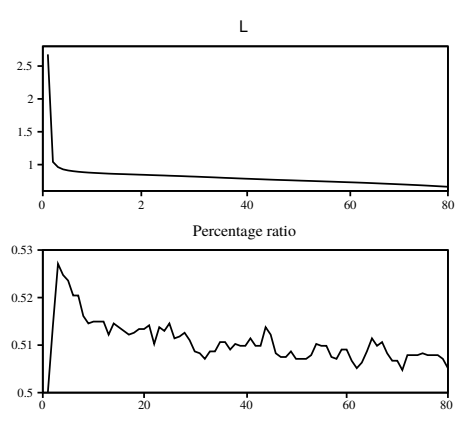

(a) Topological gradient enabled.
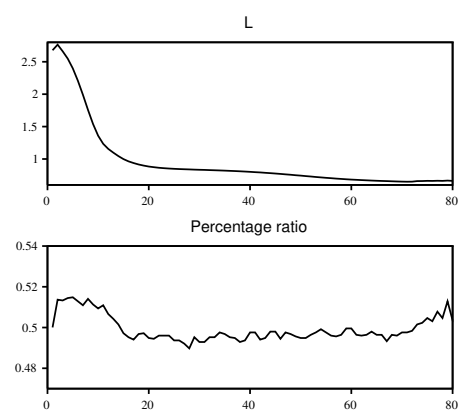

(b) Topological gradient disabled.

Fig. 10: The convergence curves for $\tau=5 \times 10^{-3}$. On the left, the topological gradient is enabled and disabled on the right. On the top, the decrease of the Lagrangian $L=J(K)\left(u_{0}\right)+\tau\left|\partial \Omega_{a}\right|$ with the number of iterations is shown and on the bottom, the respective control curve for the resource constraint volume ratio $\theta^{0}=50 \%$.

Figure 10 shows typical convergence diagrams for the objective function, $\mathcal{L}=$ $J(K)\left(u_{0}\right)+\tau\left|\partial \Omega_{a}\right|$, with perimeter penalization. The volume constraint is respected: the percentage ratio of material A oscillates within an acceptable range of $2 \%$ around the constraint value, $\theta^{0}=50 \%$. Optimal in-plane material distributions for the minimization of run-in wear volume loss are shown on Figures 8 (topological gradient disabled) and 9 (topological gradient enabled). Several material configurations are presented for cases with increasingly severe levels of perimeter penalization $\left(\tau=1.5 \times 10^{-4}, 5 \times 10^{-3}, 1.5 \times 10^{-2}\right)$ : (i) the initial material distribution, (ii) three intermediate profiles, (iii) the final unit-cell design obtained by the minimization algorithm, and (iv) a $3 \times 3$ tiling of the periodic unit-cell for ease of visualization. The values of the objective function at convergence for all of the cases shown in Figures 8 and 9 are presented in Table 1.

\begin{tabular}{|l|l|l|l|}
\hline $\begin{array}{l}\text { Topological gradi- } \\
\text { ent }\end{array}$ & $\begin{array}{l}\text { Perimeter penal- } \\
\text { ization }(\tau)\end{array}$ & $\begin{array}{l}\text { Objective function } \\
\left(\text { inf } J(K)\left(u_{0}\right)\right)\end{array}$ & $\begin{array}{l}\text { Final objective as } \\
\text { percentage of the } \\
\text { initial design }\end{array}$ \\
\hline \hline Enabled & $1.5 \times 10^{-4}$ & 0.15 & $5.8 \%$ \\
\hline Disabled & $1.5 \times 10^{-4}$ & 0.16 & $6.2 \%$ \\
\hline \hline Enabled & $5 \times 10^{-3}$ & 0.28 & $11 \%$ \\
\hline Disabled & $5 \times 10^{-3}$ & 0.39 & $15 \%$ \\
\hline \hline Enabled & $1.5 \times 10^{-2}$ & 0.68 & $26 \%$ \\
\hline Disabled & $1.5 \times 10^{-2}$ & 0.74 & $29 \%$ \\
\hline
\end{tabular}

Table 1: Optimal values for the run-in wear volume, $J(K)\left(u_{0}\right)$, depending on the perimeter penalization, $\tau$, and the use of the topological gradient, for a fixed percentage ratio $\theta^{0}=50 \%$ between the two materials. The run-in wear value for the initial design was $J(K)\left(u_{0}\right)=2.58$. 
These results show that increasing the value of the perimeter penalization coefficient, $\tau$, allows one to reduce the complexity of the final design, although at the expense of increasing the run-in wear volume loss. In addition to perimeter penalization, the shape of the final design can also be influenced by two separate factors (i) the choice of the initial configuration and (ii) by enabling or disabling the topological gradient. If the topological gradient is disabled, Figure 8 demonstrates that the level-set method still allows interface collisions to affect dramatic topological changes.

One of the useful applications of this method for wear optimization, is to improve upon existing composite designs from tribology literature. Table 11 shows that the initial guess, a matrix of material $A$ with a circular inclusion filled with material $B$ (a typical intuitive guess for designing composites), can be significantly improved upon. The run-in-wear volume lost decreased by more than $70 \%$ in all of the cases studied, including those with the highest perimeter penalization $\left(\tau=1.5 \times 10^{-2}\right)$. The results also indicate that, in comparison to the calculated steady-state profiles for the initial configurations, the optimized steady-state profiles are significantly more flat; this is according to the measures described in Section 4.2. In the periodic unit-cell framework, the numerical examples in Figures 8 and 9 illustrate that topology optimization may be used to design manufacturable periodic composites with improved wear performance and controlled complexity at the composite meso-or-microstructural scale.

The optimization of bulk tribological systems at a macroscopic structural scale can be achieved by replacing the periodic boundary conditions with nonhomogeneous Fourier conditions (equation 7) and by replacing the constant pressure profile, $P_{0}$, with a generic profile, $f$. The formulation presented here is not restricted to the specific relationship between pressure and wear profile (equation 6) used in Section 2. The existence of a steady-state solution and the expressions for the shape derivatives obtained in Section 4 are valid for any $H^{1}(\Omega)$ continuous bilinear form, $a$, coercive on $V_{K}$ and null on the constants (Feppon et al (2015)). This implies that some changes in the relationship between the pressure distribution, $p$, and the profile, $z$ (equation 6), can be taken into account without modification of the theory. For example, an additional convective term of the form $\nabla z \cdot \mathbf{u}$, for an appropriate vector field, $\mathbf{u}$, could be used to include a dependence of the pressure distribution on the direction of sliding. Certainly, more general operators beyond the Laplacian, could also be used. These topics are to be the subject of future work.

As an illustration of the utility of the formulation for bulk macroscopic structures, the model is calibrated with experimental data. In Rowe et al (2014), an experimental steady-state surface profile was obtained through the abrasive wear of a bi-material distribution of nine equally spaced inclusions (Figure 11). The experimental parameters were $L=20 \mathrm{~mm}, H=19.8 \mathrm{~mm}, K_{a}=2.24 \times 10^{-1} \mathrm{~mm}^{3} / \mathrm{Nm}$ (in black), $K_{b}=1.98 \times 10^{-2} \mathrm{~mm}^{3} / \mathrm{Nm}$ (in white), $k_{s}=3.07 \times 10^{-1} \mathrm{~N} / \mathrm{mm}^{3}, k_{g}=$ $2.8 \mathrm{~N} / \mathrm{mm}$, and $P_{0}=<f>=0.083 \mathrm{MPa}$. Figure 12 presents the experimental steady-state surface measured by a profilometer as well as a smoother version of the data with noise reduction (using a Fast Fourier Transform technique (Duhamel and Vetterli (1990))). For each of the 3D profiles shown, an additional plot along a line scan $y=4.8 \mathrm{~mm}$ is also included for ease of comparison.

Figure 13a presents the profile that was predicted in Rowe et al (2014), using a uniform pressure profile, $f=P_{0}$. In order to match the experimental data, the 


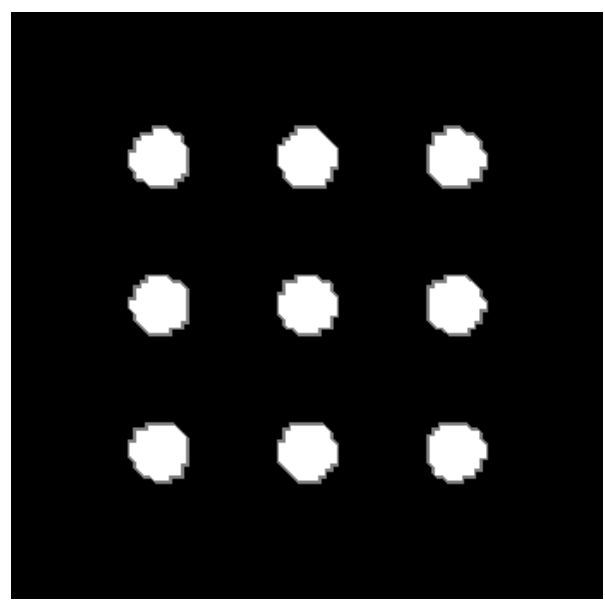

Fig. 11: Material distribution considered by Rowe et al (2014).
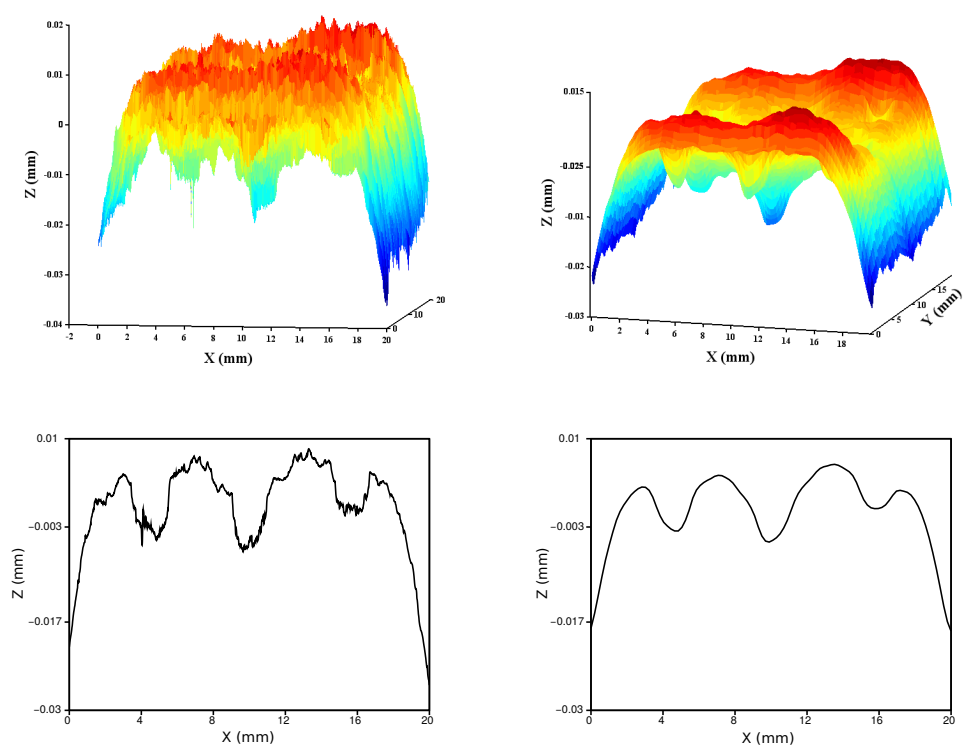

Fig. 12: On the left: experimental profile measured in Rowe et al (2014). On the right: smoothed experimental profile after noise reduction.

sensitivity of the model to the parameter $f$ has been computed and used in a gradient method (see Allaire (2007a)) to derive a non-uniform pressure profile, $f$, that results in a steady surface profile that matches the experimental data. The shape of this new reference pressure profile (Figure 14) is consistent with flat punch theory, which predicts elevated pressures at domain edges (Ciavarella et al (1998); 

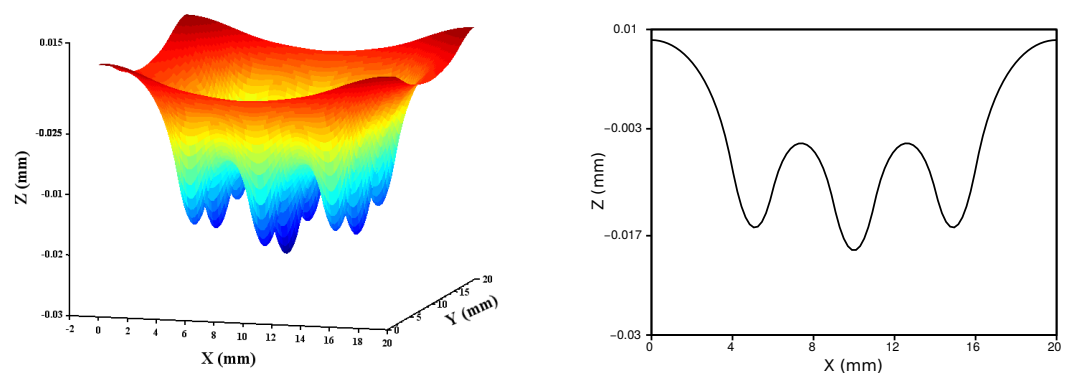

(a) Prediction of the steady-state profile using a uniform reference pressure, $f=P_{0}$.
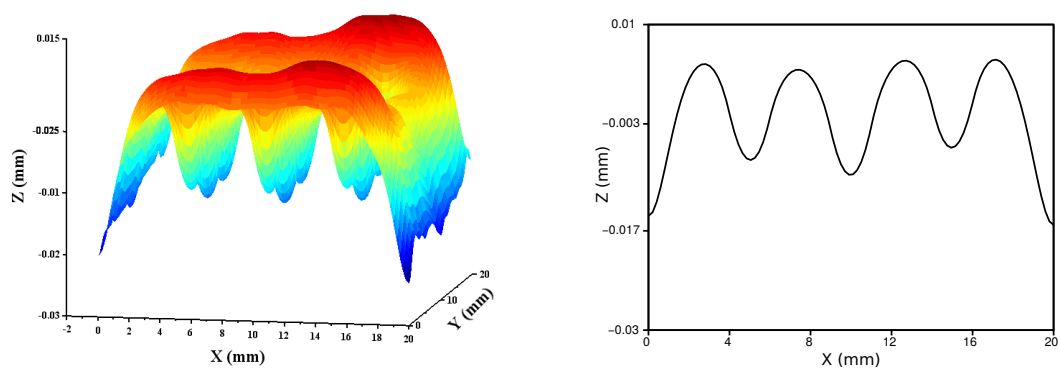

(b) Prediction of the steady-state profile using the calibrated profile of Figure 14

Fig. 13: Top: prediction of the steady state profile under Neumann or periodic boundary conditions and with a uniform pressure profile, $f=P_{0}$, as was done in Rowe et al (2014). Bottom: prediction of the steady state using the non-uniform pressure profile, $f$, of Figure 14 and Neumann boundary conditions.

Marzeda et al (2001)) (Figure 5). Figure 13b shows the steady-state surface profile that is predicted by the model with this non-uniform pressure profile.

This methodology demonstrates that the formulation presented can be calibrated with experimental data, and optimized material configurations can be identified for specific experimental set-ups. However, additional modifications of the proposed model must be considered. Close attention must be paid to specifying experimentally relevant boundary conditions. The example considered by Rowe et al (2014) shows that periodic or homogeneous Neumann boundary conditions combined with a uniform pressure profile, $f=P_{0}$, are not able to capture the rounded features found at sample edges of experimentally determined steady-state wear profiles. On the other hand, inhomogeneous Neumann boundary conditions with a source term $(g \neq 0$ described in equation $(7))$ may not be physically relevant because they must apply throughout the entire wear process, including the initial conditions where the surface may be flat and $g=0$. Furthermore, considering a non-uniform pressure profile, $f$, may not be sufficient to obtain acceptable predictions, since this profile may depend itself on the material distribution, $K$ (it has 

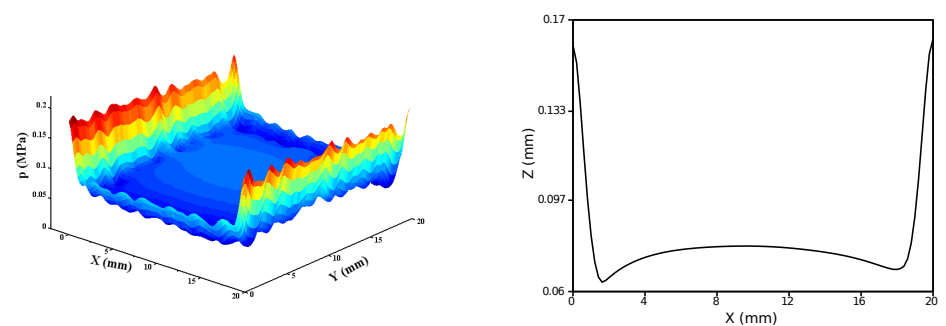

Fig. 14: Calibrated pressure profile to match the experimental data of Figure 12

been observed numerically that the example calibrated pressure profile does not result in rounded edges when changes in the material distribution occur near the boundary). Future work could focus on proposing models for the behavior of this pressure profile parameter with respect to experimentally relevant constraints.

Finally, the optimization method proposed in this article is also relevant for broader applications of topology optimization beyond wear performance. Whenever the topological gradient is an extension of the velocity of the shape derivative of the interface, $\partial \Omega_{a}$, the method offers substantial advantages related to imposing equality constraints (with affine relationships to the volume), controlling feature complexity, and topological sensitivity. The key ingredient of the proposed approach is the constraint (equation 50 on the level-set function. This constraint allows one to obtain an evolution equation for the interface that is linear and it also allows one to use the topological gradient beyond the interface zone (at distances greater than $\epsilon$ from the zero level-set).

\section{Conclusion}

A framework has been presented to optimize the performance of multimaterial composites subject to sliding abrasive wear. The optimization focuses on the steady-state wear profile which is characterized by an elliptic partial differential equation. The steady-state solution can be directly determined from known material distributions, allowing time-independent optimization to be applied. Common wear objectives are identified and formalized for optimization, like run-in volume loss and surface roughness. A relaxation result is presented that demonstrates limitations of scale that must be considered in the optimization scheme. This framework has been applied to the wear optimization of a bi-material periodic unit-cell (representing the meso-or-microstructural design of composite materials). Numerical examples demonstrate the reduction of material loss made possible by designs guided by topology optimization. They also illustrate how target steady-state wear surface roughness may be achieved. Initial results and perspectives for application of the proposed scheme to macroscopic structural design without periodic boundary conditions are provided. Several approaches to control the complexity of designs are proposed in order to respect the scale limitations of the underlying physical wear model in the optimization scheme. Broader applications of these con- 
trol schemes for other types of problems, beyond wear, in topology optimization are discussed.

Acknowledgements This material is based upon work supported by the National Science Foundation under Grant No. 1538125. This work was also funded, in part, by the Lehigh University Faculty Innovation Grant. The authors would like to acknowledge that G. Allaire is a member of the DEFI project at INRIA Saclay Ile-de-France.

\section{References}

Alexandersen J, Lazarov BS (2015) Topology optimisation of manufacturable microstructural details without length scale separation using a spectral coarse basis preconditioner. Computer Methods in Applied Mechanics and Engineering 290:156-182

Allaire G (2002) Shape Optimization by the Homogenization Method, Applied Mathematical Sciences, vol 24. Springer

Allaire G (2007a) Conception optimale de structures, vol 58. Springer. Collection : Mathématiques et Applications

Allaire G (2007b) Numerical Analysis and Optimization. An Introduction to Mathematical Modelling and Numerical Simulation, Numerical Mathematics and Scientific Computation, vol 87. OUP Oxford

Allaire G, Jouve F (2006) Coupling the level set method and the topological gradient in structural optimization. In: Bendsøe MP, Olhoff N, Sigmund O (eds) IUTAM Symposium on Topological Design Optimization of Structures, Machines and Materials, Springer Netherlands, Dordrecht, Solid Mechanics and Its Applications, vol 137, pp 3-12

Allaire G, Jouve F, Toader A (2004) Structural optimization using sensitivity analysis and a level-set method. Journal of Computational Physics 194(1):363-393

Allaire G, De Gournay F, Jouve F, Toader A (2005) Structural optimization using topological and shape sensitivity via a level set method. Control and cybernetics 34(1):59-80

Allaire G, Dapogny C, Delgado G, Michailidis G (2014a) Multi-phase structural optimization via a level set method. ESAIM: COCV 20(2):576-611

Allaire G, Jouve F, Michailidis G (2014b) Thickness control in structural optimization via a level set method. HAL preprint: hal-00985000v1, to appear in Structural and Multidisciplinary Optimization

Ambrosio L, Buttazzo G (1993) An optimal design problem with perimeter penalization. Calculus of Variations and Partial Differential Equations 1(1):55-69

Archard JF (1953) Contact and rubbing of flat surfaces. Journal of Applied Physics 24(8):981988

Archard JF, Hirst W (1956) The wear of metals under unlubricated conditions. Proceedings of the Royal Society A: Mathematical, Physical and Engineering Sciences 236(1206):397-410

ASTM Standard D3702-94 (1974/2014) Standard test method for wear rate and coefficient of friction of materials in self-lubricated rubbing contact using a thrust washer testing machine

ASTM Standard G77-05 (1983/2010) Standard test method for ranking resistance of materials to sliding wear using block-on-ring wear test

ASTM Standard G99-05 (1990/2010) Standard test method for wear testing with a pin-on-disk apparatus

Axén N, Jacobson S (1994) A model for the abrasive wear resistance of multiphase materials. Wear 174(1-2):187-199

Barron RF (1982) Cryogenic treatment of metals to improve wear resistance. Cryogenics 22(8):409-413

Bendsøe MP, Kikuchi N (1988) Generating optimal topologies in structural design using a homogenization method 71(2):197-224

Bendsøe MP, Sigmund O (2003) Topology optimization: theory, methods and applications. Springer, Berlin

Bertsekas DP (1996) Constrained optimization and Lagrange multiplier methods. Belmont, Mass. : Athena Scientific

Blanchet TA (1997) The interaction of wear and dynamics of a simple mechanism. Journal of Tribology 119(3):597 
Blau PJ (1997) Fifty years of research on the wear of metals. Tribology International 30(5):321331

Céa J (1986) Conception optimale ou identification de formes, calcul rapide de la dérivée directionnelle de la fonction coût. RAIRO-Modélisation mathématique et analyse numérique 20:371-402

Céa J, Garreau S, Guillaume P, Masmoudi M (2000) The shape and topological optimizations connection. Computer methods in applied mechanics and engineering 188(4):713-726

Challis VJ, Roberts AP, Wilkins AH (2008) Fracture resistance via topology optimization. Structural and Multidisciplinary Optimization 36(3):263-271

Chattopadhyay R (2001) Surface wear: analysis, treatment, and prevention. ASM international

Chavel I (2010) Riemannian Geometry, A Modern Introduction, vol 119. Cambridge University Press, Cambridge

Chen J, Shapiro V, Suresh K, Tsukanov I (2007) Shape optimization with topological changes and parametric control. International journal for numerical methods in engineering 71(3):313-346

Choi HY, Lee DH, Lee J (2013) Optimization of a railway wheel profile to minimize flange wear and surface fatigue. Wear 300(1-2):225-233

Chongyi C, Chengguo W, Ying J (2010) Study on numerical method to predict wheel/rail profile evolution due to wear. Wear 269(3-4):167-173

Ciavarella M, Hills D, Monno G (1998) The influence of rounded edges on indentation by a flat punch. Proceedings of the Institution of Mechanical Engineers, Part C: Journal of Mechanical Engineering Science 212(4):319-327

Colbert RS, Krick BA, Dunn AC, Vail JR, Argibay N, Sawyer WG (2011) Uncertainty in pinon-disk wear volume measurements using surface scanning techniques. Tribology Letters 42(1):129-131, DOI 10.1007/s11249-010-9744-8

Dapogny C (2013) Shape optimization, level set methods on unstructured meshes and mesh evolution. PhD thesis, École Doctorale Paris Centre

Delfour M, Zolésio J (2001) Shapes and geometries, vol 4. Analysis, differential calculus and optimization, SIAM, Philadelphia

Dickrell DJ, Sawyer WG (2004) Evolution of wear in a two-dimensional bushing. Tribology Transactions 47(2):257-262

Dickrell DJ, Dooner DB, Sawyer WG (2003) The evolution of geometry for a wearing circular cam: Analytical and computer simulation with comparison to experiment. Journal of Tribology 125(1):187

Duhamel P, Vetterli M (1990) Fast fourier transforms: A tutorial review and a state of the art. Signal Processing 19(4):259-299

Erickson GM, Sidebottom MA, Curry JF, Kay D, Kuhn-Hendricks SM, Norell MA, Sawyer WG, Krick BA (2015) Paleo-tribology: Development of wear measurement techniques and a three-dimensional model revealing how grinding dentitions self-wear to enable functionality. Surface Topography: Metrology and Properties Special Issue Article

Eschenauer HA, Olhoff N (2001) Topology optimization of continuum structures: A review. Applied Mechanics Reviews 54(4):331

Feppon F, Michailidis G, Sidebottom M, Krick B, Vermaak N (2015) Efficient steady-state computation for wearing of multi-material composites. Preprint submitted to ASME Journal of tribology

Fregly BJ, Sawyer WG, Harman MK, Banks SA (2005) Computational wear prediction of a total knee replacement from in vivo kinematics. Journal of Biomechanics 38(2):305-314

Gomes J, Faugeras O (1999) Reconciling distance functions and level sets. Scale-Space Theories in Computer Vision, Springer pp 70-81

Guest JK, Prévost JH, Belytschko T (2004) Achieving minimum length scale in topology optimization using nodal design variables and projection functions. International Journal for Numerical Methods in Engineering 61(2):238-254

Han SW, Blanchet TA (1997) Experimental evaluation of a steady-state model for the wear of particle-filled polymer composite materials. Journal of tribology 119(4):694-699

Hatchett C (1803) Experiments and observations on the various alloys, on the specific gravity, and on the comparative wear of gold. being the substance of a report made to the right honourable the lords of the committee of privy council, appointed to take into consideratio. Philosophical Transactions of the Royal Society of London 93(0):43-194

He L, Kao CY, Osher S (2007) Incorporating topological derivatives into shape derivatives based level set methods. Journal of Computational Physics 225(1):891-909 
Hecht F (2012) New development in freefem++. Journal of Numerical Mathematics 20(34):251-266

Hilding D, Klarbring A, Petersson J (1995) Optimization of structures in unilateral contact. ASME Appl Mech Rev 52(397)

Hilding D, Torstenfelt B, Klarbring A (2001) A computational methodology for shape optimization of structures in frictionless contact. Computer Methods in Applied Mechanics and Engineering 190(31):4043-4060

Hirst W, Lancaster JK (1956) Surface film formation and metallic wear. Journal of Applied Physics 27(9):1057-1065

Hovis SK, Talia JE, Scattergood RO (1986) Erosion in multiphase systems. Wear 108(2):139155

Jang I, Burris DL, Dickrell PL, Barry PR, Santos C, Perry SS, Phillpot SR, Sinnott SB, Sawyer WG (2007) Sliding orientation effects on the tribological properties of polytetrafluoroethylene. Journal of Applied Physics 102(12):123,509

Jansen M, Lazarov BS, Schevenels M, Sigmund O (2013) On the similarities between micro/nano lithography and topology optimization projection methods. Structural and Multidisciplinary Optimization 48(4):717-730

Johansson L (1994) Numerical simulation of contact pressure evolution in fretting. Journal of tribology 116(2):247-254

Kerr AD (1964) Elastic and viscoelastic foundation models. Journal of Applied Mechanics $31(3): 491$

Khruschov M (1974) Principles of abrasive wear. Wear 28(1):69-88

Kim NH, Won D, Burris D, Holtkamp B, Gessel GR, Swanson P, Sawyer WG (2005) Finite element analysis and experiments of metal/metal wear in oscillatory contacts. Wear 258(1112):1787-1793

Lancaster J (1969) Abrasive wear of polymers. Wear 14(4):223-239

Lee GY, Dharan CKH, Ritchie RO (2002) A physically-based abrasive wear model for composite materials. Wear 252(3-4):322-331

Lengiewicz J, Stupkiewicz S (2013) Efficient model of evolution of wear in quasi-steady-state sliding contacts. Wear 303(1-2):611-621

Luo J, Luo Z, Chen S, Tong L, Wang MY (2008) A new level set method for systematic design of hinge-free compliant mechanisms. Computer Methods in Applied Mechanics and Engineering 198(2):318-331

Markine VL, Shevtsov IY, Esveld C (2007) An inverse shape design method for railway wheel profiles. Structural and Multidisciplinary Optimization 33(3):243-253

Marzeda J, Pauk V, Woźniak M (2001) Contact of a rigid flat punch with a wedge supported by the winkler foundation. Journal of Theoretical and Applied Mathematics

Meisel N, Gaynor A, Williams C, Guest J (2013) Multiple-material topology optimization of compliant mechanisms created via polyjet 3d printing. In: 24th Annual international solid freeform fabrication symposium an additive manufacturing conference

Mukras S, Kim NH, Sawyer WG, Jackson DB, Bergquist LW (2009) Numerical integration schemes and parallel computation for wear prediction using finite element method. Wear 266(7-8):822-831

Mukras S, Kim NH, Mauntler NA, Schmitz TL, Sawyer WG (2010) Analysis of planar multibody systems with revolute joint wear. Wear 268(5-6):643-652

Murat F, Tartar L (1985) Calcul des variations et homogénéisation. Coll. Dir. Études et Recherches EDF, 57, Eyrolles, Paris

Myśliński A (2012) Topology optimization of quasistatic contact problems. International Journal of Applied Mathematics and Computer Science 22(2):269-280

Neittaanmäki P, Sokolowski J, Zolesio JP (1988) Optimization of the domain in elliptic variational inequalities. Applied mathematics \& optimization 18(1):85-98

Osher S, Fedkiw R (2004) Level set methods and dynamic implicit surfaces. In: Applied Mechanics Reviews, vol 57, p B15

Osher S, Sethian J (1988) Fronts propagating with curvature-dependent speed: algorithms based on hamilton-jacobi formulations. Journal of computational physics 79(1):12-49

Põdra P, Andersson S (1997) Wear simulation with the winkler surface model. Wear 207(12):79-85

Põdra P, Andersson S (1999a) Finite element analysis wear simulation of a conical spinning contact considering surface topography. Wear 224(1):13-21 
Põdra P, Andersson S (1999b) Simulating sliding wear with finite element method. Tribology International $32(2): 71-81$

Páczelt I, Mróz Z (2005) On optimal contact shapes generated by wear. International Journal for Numerical Methods in Engineering 63(9):1250-1287

Pasternak PL (1954) On a new method of analysis of an elastic foundation by means of two foundation constants. Gosudarstvennoe Izdatel'stvo Litearturi po Stroitel'stvu i Arkhitekture, Moscow, USSR (in Russian)

Prasad BK (2000) Effect of microstructure on the sliding wear performance of a zn-al-ni alloy. Wear 240(1):100-112

Rabinowicz E, Mutis A (1965) Effect of abrasive particle size on wear. Wear 8(5):381-390

Rabinowicz E, Dunn L, Russell P (1961) A study of abrasive wear under three-body conditions. Wear 4(5):345-355

Rowe KG, Erickson GM, Sawyer WG, Krick BA (2014) Evolution in surfaces: Interaction of topography with contact pressure during wear of composites including dinosaur dentition. Tribology Letters 54(3):249-255

Sawyer WG (2001) Wear predictions for a simple-cam including the coupled evolution of wear and load. Lubr Eng 57(9):31-36

Sawyer WG (2004) Surface shape and contact pressure evolution in two component surfaces: Application to copper chemical mechanical polishing. Tribology Letters 17(2):139-145

Sawyer WG, Argibay N, Burris DL, Krick BA (2014) Mechanistic studies in friction and wear of bulk materials. Annual Review of Materials Research 44(1):395-427

Schevenels M, Lazarov BS, Sigmund O (2011) Robust topology optimization accounting for spatially varying manufacturing errors. Computer Methods in Applied Mechanics and Engineering 200(49):3613-3627

Schmidt HB, Hattel JH (2008) Thermal modelling of friction stir welding. Scripta Materialia $58(5): 332-337$

Schmitz TL, Action JE, Burris DL, Ziegert JC, Sawyer WG (2004) Wear-Rate Uncertainty Analysis. Journal of Tribology 126(4):802, DOI 10.1115/1.1792675

Schury F, Stingl M, Wein F (2012) Efficient two-scale optimization of manufacturable graded structures. SIAM Journal on Scientific Computing 34(6):B711-B733

Seepersad CC, Allen JK, McDowell DL, Mistree F (2006) Robust design of cellular materials with topological and dimensional imperfections. Journal of Mechanical Design 128(6):1285-1297

Sidebottom MA, Feppon F, Vermaak N, Krick BA (2015) Modelling wear of multi-material composite wear surfaces. ASME Journal of Tribology Submitted

Sigmund O (2009) Manufacturing tolerant topology optimization. Acta Mechanica Sinica $25(2): 227-239$

Sin H, Saka N, Suh N (1979) Abrasive wear mechanisms and the grit size effect. Wear 55(1):163190

Sokolowski J, Z̃ochowski A (1999) On the topological derivative in shape optimization. SIAM J Control Optim 37:1251-1272

Strömberg N, Klarbring A (2010) Topology optimization of structures in unilateral contact. Structural and Multidisciplinary Optimization 41(1):57-64

Tankala K, Kapoor R (2001) Method for improving wear resistance of abrasive tools US Patent $6,227,188$

Tartar L (2009) The General Theory of Homogenization: A Personalized Introduction. Lecture Notes of the Unione Matematica Italiana, Berlin Heidelberg

Telliskivi T (2004) Simulation of wear in a rolling-sliding contact by asemi-winkler model and the archard's wear law. Wear 256(7-8):817-831

Vermaak N, Michailidis G, Parry G, Estevez R, Allaire G, Bréchet Y (2014) Material interface effects on the topology optimizationof multi-phase structures using a level set method. Structural and Multidisciplinary Optimization 50(4):623-644

Wang F, Lazarov BS, Sigmund O (2011) On projection methods, convergence and robust formulations in topology optimization. Structural and Multidisciplinary Optimization 43(6):767-784

Wang MY, Wang X, Guo D (2003) A level set method for structural topology optimization. Computer Methods in Applied Mechanics and Engineering 192(1-2):227-246

Willing R, Kim IY (2009) Three dimensional shape optimization of total knee replacements for reduced wear. Structural and Multidisciplinary Optimization 38(4):405-414 
Yamada T, Izui K, Nishiwaki S, Takezawa A (2010) A topology optimization method based on the level set method incorporating a fictitious interface energy. Computer Methods in Applied Mechanics and Engineering 199(45-48):2876-2891

Zhou M, Lazarov BS, Sigmund O (2014) Topology optimization for optical projection lithography with manufacturing uncertainties. Applied optics 53(12):2720-2729

Zmitrowicz A (2006) Wear patterns and laws of wear - a review. Theoretical And Applied Mechanics 44(1803):219-253

Zum Gahr KH (1987) Microstructure and wear of materials, vol 10. Amsterdam; New York; Elsevier 